\title{
Stability analysis of high-order finite-difference discretizations of the linearized
} forward-speed seakeeping problem

\author{
Amini-Afshar, Mostafa; Bingham, Harry B.; Henshaw, William D.
}

\section{Published in:}

Applied Ocean Research

Link to article, DOI:

10.1016/j.apor.2019.101913

Publication date:

2019

Document Version

Peer reviewed version

Link back to DTU Orbit

Citation (APA):

Amini-Afshar, M., Bingham, H. B., \& Henshaw, W. D. (2019). Stability analysis of high-order finite-difference discretizations of the linearized forward-speed seakeeping problem. Applied Ocean Research, 92, [101913]. https://doi.org/10.1016/j.apor.2019.101913

\section{General rights}

Copyright and moral rights for the publications made accessible in the public portal are retained by the authors and/or other copyright owners and it is a condition of accessing publications that users recognise and abide by the legal requirements associated with these rights.

- Users may download and print one copy of any publication from the public portal for the purpose of private study or research.

- You may not further distribute the material or use it for any profit-making activity or commercial gain

- You may freely distribute the URL identifying the publication in the public portal 


\title{
Stability analysis of high-order finite-difference discretizations of the linearized forward-speed seakeeping problem
}

\author{
Mostafa Amini-Afshar ${ }^{1}$, Harry B. Bingham ${ }^{1}$, and William D. Henshaw ${ }^{2}$ \\ ${ }^{1}$ Department of Mechanical Engineering, Technical University of Denmark \\ Section for Fluid Mechanics, Coastal and Maritime Engineering \\ maaf@mek . dtu . dk, hbb@mek . dtu . dk \\ ${ }^{2}$ Department of Mathematical Sciences, Rensselaer Polytechnic Institute, New York \\ henshw@rpi.edu
}

\begin{abstract}
A high-order finite-difference method solution of the linearized, potential flow, seakeeping problem for a ship at steady forward speed was recently presented by Amini-Afshar et al $[1,2]$. In this paper, we provide a detailed matrix-based eigenvalue stability analysis of this model, highlighting the sources of instability and the effects of possible remedies. In particular, we illustrate how both boundary treatment and grid stretching are important factors which are not typically captured by a von Neumann-type analysis. The new analysis shows that when grid stretching is used together with centered finite difference schemes, the method is generally unstable. The source of the instability can in some cases be traced to an effective downwinding of the convective terms. Stable solutions can be obtained either by introducing upwind-biased schemes for computing the convective derivatives on the free-surface, or by application of a mild filter at each time-step. A second source of instability is associated with the treatment of the convective derivatives of the free-surface elevation at points close to the domain boundaries. Here it is necessary to consider whether the surrounding fluid points lie in an upwind or a downwind direction. For upwinded points, ordinary one-sided differencing can be used, but for downwinded points we instead impose a Neumann-type boundary condition derived from the body and free-surface boundary conditions. As an example application to complement those already given in $[1,2]$, the method is applied to solve the steady wave resistance problem and comparison is made to reference solutions for a two-dimensional floating cylinder and a submerged sphere. Estimates of the wave resistance of the Wigley hull are also compared with experimental measurements.
\end{abstract}

\section{Keywords}

Stability analysis, Wave resistance, Neumann-Kelvin linearization, Finite difference, Overlapping grids, Forward speed

\section{Introduction}

An accurate prediction of the steady wave resistance of a ship is important for estimating the required propulsion power and optimizing the design of the hull form. In the past 30 years or so, a large number of numerical methods have been developed for solving this problem, based either on the assumption of a potential flow or the Navier-Stokes equations (CFD). CFD solutions are now quite mature and reliable for solving the ship resistance problem, and can thus be considered to be the state of the art, see for example [3], [4] and [5].

A complete analysis of the ship however, requires a prediction of the unsteady response in waves (the seakeeping problem) and here the computational cost of a CFD analysis is still prohibitive. Thus, 
solutions based on the assumption of a potential flow are still highly relevant. Moreover the linearization of the seakeeping problem can be valid and reasonable as long as the underlying assumptions for the associated linear hydrodynamic solutions are satisfied. Specifically this implies that linearized solutions are reliable as long as the body's motions are small relative to its length scale(s), and the amplitudes of both the ship generated waves and the incident waves are well bellow the breaking limit, so that small-amplitude theory is a good approximation. With regard to computational effort and desired accuracy, linearized hydrodynamics provides efficient solutions for small-amplitude seakeeping problems. The seakeeping problem is generally linearized about the steady flow solution, so in this context it is important to be able to solve the steady problem accurately with a potential flow solver. Potential flow solutions of the wave resistance problem are most commonly based on the Boundary Element Method (BEM), see for example [6], [7], [8], [9], [10] and [11]. Some of these authors also presented von Neumann-type stability analyses of the methods where the domain is assumed to be infinite (periodic) and the grid spacing uniform and rectangular, thus the effects of curved boundaries and irregular grids were not included.

Over the past ten years, a computational strategy has been developed at the Technical University of Denmark for efficient and accurate simulation of nonlinear water waves [12],[13]. A Method of Lines approach [14] is adopted, where the spatial and temporal discretizations are done separately. The explicit fourth-order, four-stage Runge-Kutta time integration scheme is invoked in time, and arbitrary-order finite-difference schemes are applied in space to discretize the Laplace equation and boundary conditions. Compared to the BEM method, this approach requires an order of magnitude more grid points, since the full 3D computational domain needs to be discretized, but the sparse nature of the resultant linear system of equations allows for an optimum scaling of the computational effort with increasing resolution. This combined with the high-order accuracy of the discrete operators makes the approach competitive with existing BEM solutions, especially for nonlinear problems.

The application of this strategy to solving the forward-speed seakeeping problem was presented in $[1,2]$. Here the details of the basic solution strategy were described and demonstrations of both convergence and the scaling of the solution effort were provided. Application examples focused on the radiation and the diffraction problems respectively for both academic and real ship geometries. In this paper, we present a detailed stability analysis of the scheme and present solutions to the steady wave resistance problem.

In the context of a method of lines solution, the stability of the numerical solution can be analyzed by forming the spatial Jacobian matrix of the semi-discrete system on a given grid, and comparing the eigenvalues (scaled by the time step size) with the stability region of the selected time integration method [14]. Although this analysis can only be carried out using relatively small numbers of grid points, it should give an accurate picture of the behavior of the full solution in general. In contrast to a von Neumann-type analysis, this matrix-based stability analysis allows us to investigate the influence of boundary conditions, curvilinear and stretched grids and combinations of centered and off-centered finite-difference stencils on the stability of the numerical method.

The stability analysis performed here shows that grid stretching can destabilize the numerical method if a centered stencil is employed to calculate the convective derivatives. This is explained by the fact that the finite-difference coefficients increase as the grid spacing reduces, weighting the closer points more strongly than those further away, which effectively downwinds the scheme when the grid spacing increases in the upstream direction. This has been shown in an earlier work by [15], where the spacial discretization was performed in the physical domain. In this paper, it is also shown that the same behavior occurs when the discretization is performed in a unit-spaced rectangular mapped domain. Although the instability cannot, in this case, be directly traced to a down-winding bias, it is clearly visible in the eigenvalues of the Jacobian. Two methods are proposed to stabilize the solution. The first is to apply an upwind-biased stencil to calculate the convective derivatives. This will increase the weight of the coefficients on the upwind side and stabilize the numerical method. Stability is indicated by finding all stability eigenvalues inside the stability region of the selected time integration scheme.Also apparent from the eigenvalues is the amount of numerical diffusion introduced 
by the scheme, which is indicated by their distance from the imaginary axis. A second remedy is thus to directly introduce numerical diffusion by simply applying a polynomial filter along with centered non-diffusive convective schemes.

An additional, and related, source of instability comes from the evaluation of the convective derivative of the free-surface elevation near the solid boundaries of the domain. Here, one-sided schemes can be used when they are upwinded, but there will always be locations where they are in fact downwinded, which will again lead to instability. To avoid this, we derive a Neumann boundary condition from the free-surface and body boundary conditions for application to downwinded regions. This combination of remedies is found to give stable and robust solutions of linear forward speed hydrodynamic problems using a high-order finite-difference method.

This introduction is followed by a review of the mathematical formulation for the wave resistance problem. In section 3, the numerical method is described along with a brief discussion of the way the computational grids are generated for simple geometries. The matrix-based stability analysis is presented in section 4. Finally validation examples for the wave resistance of a $2 \mathrm{D}$ cylinder, a submerged sphere and the Wigley hull are presented in section 6. The hyperbolic grid generation [16] technique used to build the grid for the ship hull is also briefly explained in this section.

\section{Formulation}

In this section a brief review of the mathematical model of the wave resistance problem is presented. For more detailed presentations of the theory see for example [17]. The wave making part of the ship resistance can be well calculated by the potential flow assumption. This is justifiable as the Reynolds number defined based on the length of the ship is relatively high, which implies the low significance of the viscosity in this hydrodynamic problem. A potential flow model is considered here where the kinematics of the fluid flow can be described by the velocity potential $\phi$. An inertial Cartesian coordinate system $(\mathrm{O}-x y z)$ is adopted where $x$ points along the length of the ship, and the $z$-axis is directed positive upward through the center of the gravity of the ship. The coordinate system is in steady translation with the same forward speed $U$ as the body, and its origin rests at the undisturbed free surface. The fluid domain is bounded by the body surface $S_{B}$, the sea bed $S_{0}$, the free surface $S_{F}$ and the far-field truncation boundary $S_{\infty}$, as is illustrated in Figure 1. Having said this, the continuity

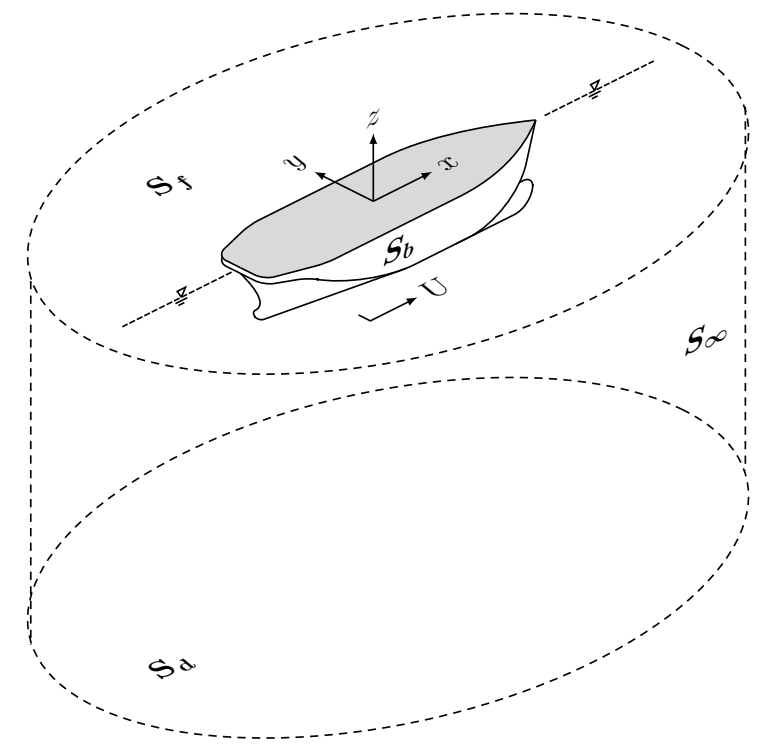

Figure 1: The coordinate system and model sketch. 
equation will be governing in the domain and is expressed by the Laplace equation as follows:

$$
\nabla^{2} \phi=\frac{\partial^{2} \phi}{\partial x^{2}}+\frac{\partial^{2} \phi}{\partial y^{2}}+\frac{\partial^{2} \phi}{\partial z^{2}}=0 .
$$

Appropriate conditions are also to be applied at the domain boundaries. The classical NeumannKelvin linearization of the exact hydrodynamic problem is adopted in this paper, see [17]. At the free surface $\boldsymbol{S}_{f}$ the linearized kinematic and dynamic conditions are applied as:

$$
\begin{aligned}
& \frac{\partial \phi}{\partial t}=-g \zeta+U \frac{\partial \phi}{\partial x}, \quad \text { at } z=0, \\
& \frac{\partial \zeta}{\partial t}=\frac{\partial \phi}{\partial z}+U \frac{\partial \zeta}{\partial x}, \quad \text { at } z=0 .
\end{aligned}
$$

The free surface elevation is denoted by $\zeta$, and $g$ is acceleration due to the gravity. The no-flux condition is applied at the mean position of the body surface $\boldsymbol{S}_{b}$, at the far-field $\boldsymbol{S}_{\infty}$, and also at the sea bed $\boldsymbol{S}_{d}$ as follows:

$$
\begin{array}{ll}
\boldsymbol{n} \cdot \nabla \phi=U n_{x}, & \text { at } \boldsymbol{S}_{b}, \\
\boldsymbol{n} \cdot \nabla \phi=0, & \text { at } \boldsymbol{S}_{\infty}, \\
\boldsymbol{n} \cdot \nabla \phi=0, & \text { at } \boldsymbol{S}_{d} .
\end{array}
$$

where $\boldsymbol{n}=\left(n_{x}, n_{y}, n_{z}\right)$ is the unit normal vector to $\boldsymbol{S}_{b}$. Note that the steady wave resistance problem in this paper is solved by considering a time dependent forward speed $\hat{U}(t)$. The forward speed is started from zero and gradually approaches the desired value $U$. The simulation is run until a nearly steady-state solution is obtained. The applied hydrodynamic force on the body can be calculated at each time step using the linearized Bernoulli equation:

$$
p=-\rho\left[\frac{\partial \phi}{\partial t}-U \frac{\partial \phi}{\partial x}\right] .
$$

Now a numerical solution to the above mentioned Neumann-Kelvin linearized hydrodynamic problem is obtained using a finite-difference discretization on overlapping grids. In the next section the numerical method used for solving the problem is outlined.

\section{$3 \quad$ Numerical methods}

A detailed description of our numerical implementation was presented in [1]. For convenience, and to provide the necessary background for the new matrix-based stability analysis presented below, a brief review of the underlying numerical methods for this solver is repeated in this section.

\subsection{Spatial and temporal discretization}

As mentioned before, the domain is bounded by the body, the sea bed, the free surface and the far-field truncation boundary. The method of lines approach is used to solve the hydrodynamic problem.

Given known initial values of $\zeta$ and $\phi(x, y, z=0)$, the Laplace equation is solved on the spatially discretized domain to give the corresponding $\phi(x, y, z)$. This allows the right-hand sides in the freesurface conditions (2.2), (2.3) to be computed. Then using the explicit four-stage, fourth-order RungeKutta scheme, the free-surface boundary conditions are marched in time to give the new values of $\zeta$ and $\phi(x, y, z=0)$. For the spacial discretization, fourth order finite-difference schemes are applied to discretize the continuous continuity equation (2.1) in the domain. The spacial discretization is performed using curvilinear body-fitted grids, each extended by two ghost layers to deal with the discretization at the boundaries. The continuity equation is applied everywhere in the overset grid 
except at the ghost, interpolation and hole points. The equations for the discretization, ghost and interpolation points are assembled into a coefficient matrix $\mathbf{A}$. The solution of the hydrodynamic problem $\mathbf{p}$ is then obtain by solving the resultant linear system of equations:

$$
\mathbf{A p}=\mathbf{b},
$$

in which the right-hand side vector $\mathbf{b}$ is defined based on the type of the equations in the coefficient matrix. The initial boundary value problem which has been presented in the previous section requires the solution of equation (3.1) and the time integration of the free-surface conditions (2.2), (2.3) at each time step throughout the simulation. More details on the discrete solution strategy can be found in $[1]$.

\subsection{Discretization of the free-surface boundary conditions}

In the case of a centered finite-difference stencil, two ghost layers should be populated at the boundaries before calculating the convective derivatives. The ghost layers can be set either by applying a Neumann-type condition at the boundary or by the extrapolation from the internal grid points. As will be shown in the next section, the correct choice for setting the ghost layers is critical to ensure a stable numerical scheme. Figure 2 shows how a combination of Neumann conditions and extrapolation are used to populate the ghost points. As shown in this figure, two regions are distinguished as follows:

$$
\begin{array}{ll}
\boldsymbol{n} \cdot \boldsymbol{W}<0 & \text { the outflow region, } \\
\boldsymbol{n} \cdot \boldsymbol{W}>0 & \text { the inflow region, }
\end{array}
$$

where $\boldsymbol{W}=(U, 0,0)$. Extrapolation in inflow regions can easily turn the centered stencil at the boundary into a downwind stencil, which makes the numerical scheme unconditionally unstable. Similarly, the hyperbolic nature of the free-surface boundary conditions (2.2) and (2.3) suggests that the inflow and outflow conditions should be consistent with the direction of the flow of information [18]. As illustrated for example by [14] using the one-dimensional advection equation, prescribing improper boundary conditions at the outflow boundary leads to spurious reflections from that location. Instead, the boundary values in this region should be obtained by extrapolation from the interior region. The same behavior is observed in the forward-speed seakeeping problem unless the choices illustrated in Figure 2 are adopted. Later in section 4 and using the matrix-based stability analysis, the influence of the Neumann and the extrapolation conditions on the numerical stability will be demonstrated.

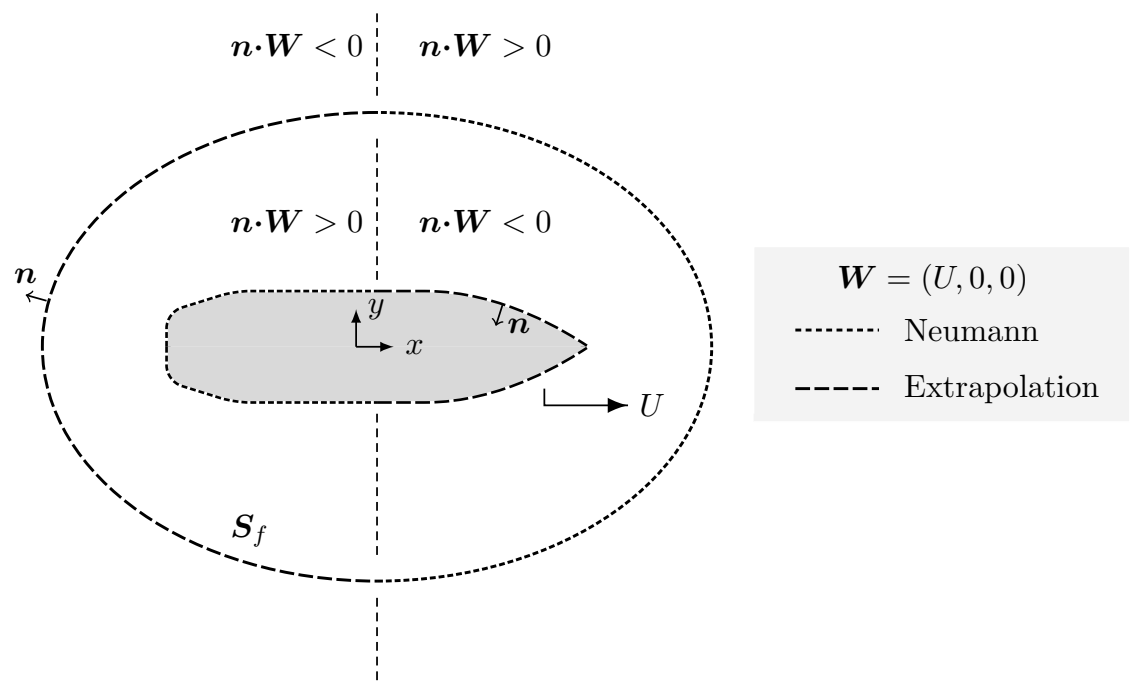

Figure 2: Inflow and outflow regions. The figure shows the regions for the extrapolation and the Neumann conditions which are used to set the ghost points before calculating the free-surface derivatives. 


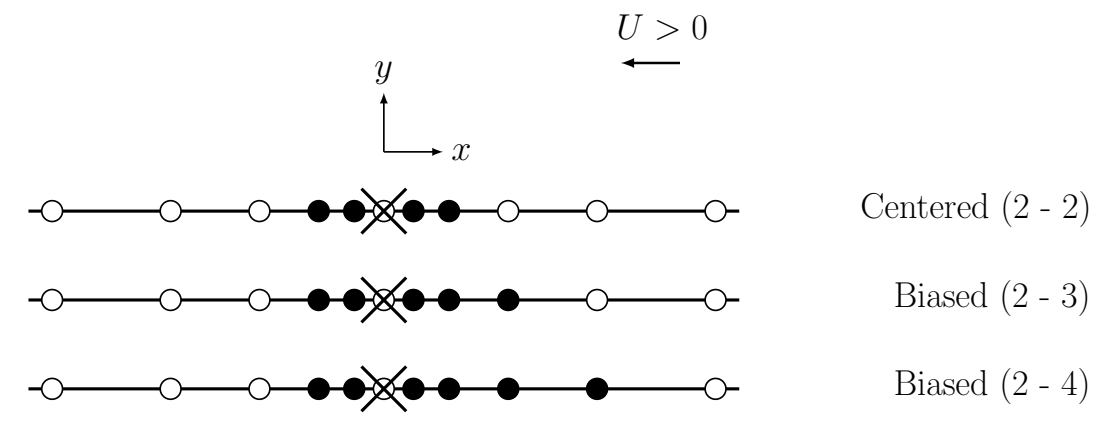

Figure 3: Illustration of the different finite-difference stencils for computing the convective derivatives on a one-dimensional stretched grid for the point marked by the cross. The original centered stencil (top), the one-point upwind-biased stencil (middle) and the two-point upwind-biased stencil (bottom). The upwind direction is determined by the positive sign of $U \Delta x$ along a grid line.

\subsubsection{Discretization of the convective derivatives}

For a uniform grid, with the aforementioned treatment of the convective derivatives at the boundaries, a stable numerical scheme can be achieved. However, as illustrated in $\S 4$, non-uniformities in the grid can easily lead to instability when solving forward-speed hydrodynamic problems.

Figure 3 illustrates some examples of one-dimensional centered and up-winded stencils for discretizing the convective derivatives on the free-surface. The plot at the top shows a centered $4^{\text {th }}$-order, 5 -point stencil while the middle plot shows an up-winded $5^{\text {th }}$-order accurate, 6 -point stencil for a ship moving from left to right. The bottom plot also shows an up-winded $6^{\text {th }}$-order accurate stencil using 7 points on the grid. Note that in three-dimensions, the appropriate upwind shift along each grid line is determined by the positive sign of $U \Delta x$, where $\Delta x$ is the change in the $x$-coordinate along the corresponding grid line.

The finite-difference weights for these schemes can be derived in the standard way via inversion of the corresponding Vandermonde matrix, or more efficiently using the recursive algorithms of [19]. The weights can be derived either in the physical domain or in a uniformly-spaced computational domain after applying a coordinate transformation.

The centered scheme can also be made stable by applying a least-squares type filter of the form:

$$
\phi^{f}\left(x_{i}\right)=\phi\left(x_{i}\right)-\sigma \sum_{j=-p}^{q} d_{j} \phi\left(x_{i}+j \Delta x\right),
$$

in which $\phi^{f}\left(x_{i}\right)$ is the solution after applying the filter. The coefficients $d_{j}$ can be obtained based on centered Savitzky-Golay filters [20] for the internal grid points. Near the boundaries, the off-centered schemes of [21] are used. The strength of the filter can be adjusted through the constant $\sigma$, and the centered filters can be of any desired order and width. Both the upwind-biased scheme and the filter have been implemented inside the developed solver [22], and either of them can be used to ensure the stability of the numerical scheme.

\section{$4 \quad$ Stability analysis}

\subsection{The semi-discrete system of linear ordinary differential equations}

In this section, we review the background for performing a matrix-based stability analysis of the above described numerical solution. Consider the dynamic and kinematic free-surface conditions (2.2) and (2.3) arranged in the following matrix form in two-dimensions

$$
\frac{\partial}{\partial t}\left[\begin{array}{l}
\zeta \\
\tilde{\phi}
\end{array}\right]=\left[\begin{array}{cc}
U \frac{\partial}{\partial x} & \frac{\partial}{\partial z} \\
-g & U \frac{\partial}{\partial x}
\end{array}\right]\left[\begin{array}{l}
\zeta \\
\tilde{\phi}
\end{array}\right],
$$


where $\zeta$ is the surface elevation and $\tilde{\phi}$ is the velocity potential at the free surface. A discretized representation of the boundary conditions can be written

$$
\frac{\partial \mathbf{f}}{\partial t}=\left[\begin{array}{cc}
U \mathbf{D}_{\mathbf{x}} & \mathbf{D}_{\mathbf{n}} \\
-g \mathbf{I} & U \mathbf{D}_{\mathbf{x}}
\end{array}\right] \mathbf{f}=\mathbf{J} \mathbf{f},
$$

The grid is taken to be rectangular with $N_{x}$ and $N_{z}$ grid points in the $x$ and $z$ directions, and the $\mathbf{f}$ vector contains the surface elevations and velocity potentials as follows:

$$
\mathbf{f}=\left[\zeta_{1} ; \zeta_{2} ; \cdots \zeta_{N_{x}} ; \tilde{\phi}_{1} ; \tilde{\phi}_{2} ; \cdots \tilde{\phi}_{N_{x}}\right]=\left[\mathbf{z}_{\mathbf{0}} ; \mathbf{p}_{\mathbf{0}}\right] .
$$

The identity matrix $\mathbf{I}$ here is of size $N_{x}$, and $\mathbf{J}$ is referred to as the Jacobian matrix. The discrete matrix operator $\mathbf{D}_{\mathbf{x}}$, also of size $N_{x}$, differentiates $\tilde{\phi}$ or $\zeta$ at each grid point with respect to $x$. This matrix will depend on the discretization strategy which has been adopted for the convective derivatives. The Dirichlet-to-Neumann operator matrix $\mathbf{D}_{\mathbf{n}}$ can be expressed in terms of the discrete Laplace problem of $(3.1)$

$$
\mathbf{A p}=\mathbf{b}=\mathbf{P}^{0} \mathbf{p}_{\mathbf{0}}
$$

where the $N$ by $N_{x}$ matrix $\mathbf{P}^{\mathbf{0}}$ has been introduced to place the free-surface values of $\tilde{\phi}$ in the correct positions of the $\mathbf{b}$ vector. Now defining the $N_{x}$ by $N_{z}$ matrix $\mathbf{D}_{\mathbf{z}}^{\mathbf{0}}$, which takes the vertical derivative of the free-surface potential, we have finally:

$$
\mathbf{D}_{\mathbf{n}}=\mathbf{D}_{\mathbf{z}}^{\mathbf{0}} \mathbf{A}^{-1} \mathbf{P}^{0}
$$

Since computing $\mathbf{D}_{\mathbf{n}}$ explicitly from (4.5) requires forming the inverse of the coefficient matrix $\boldsymbol{A}$, this task is computationally much more expensive than an ordinary solution of the system. However, for relatively small two-dimensional problems, it is quite feasible to carry this out for analysis purposes.

If the Jacobian matrix $\mathbf{J}$ is non-singular, then it has a set of independent eigenvectors $\mathbf{r}_{i}$ and according the definition of the eigenvalue problem it can be written [14]:

$$
\mathbf{J r}_{i}=\lambda_{i} \mathbf{r}_{i} \quad i=1,2, \cdots N
$$

Denoting $\mathbf{D}$ as the diagonal matrix of the eigenvalues of $\mathbf{J}$, we can also write:

$$
\mathbf{J}=\mathbf{R D R}^{-1} \quad \text { and } \quad \mathbf{D}=\mathbf{R}^{-1} \mathbf{A R}
$$

in which $\mathbf{R}=\left[\mathbf{r}_{1}, \mathbf{r}_{2} \cdots \mathbf{r}_{i}\right]$ is the matrix of the eigenvectors. If the $\mathbf{f}$ vector is transformed to the eigenvector space using $\mathbf{u}=\mathbf{R}^{-1} \mathbf{f}$, then multiplying equation (4.2) by $\mathbf{R}^{-1}$ gives:

$$
\mathbf{R}^{-1} \mathbf{f}_{t}=\left(\mathbf{R}^{-1} \mathbf{A R}\right)\left(\mathbf{R}^{-1} \mathbf{f}\right)
$$

Now a decoupled system of equations has been obtained as:

$$
\mathbf{u}_{t}=\mathbf{D u}
$$

in which $u_{t}^{p}=\lambda_{p} u$ is the $p$ th equation and can be marched separately in time by the desired integration scheme. To ensure numerical stability, the eigenvalues of the Jacobian matrix multiplied by the time step $\lambda_{p} \Delta t$, should be inside the stability region of the integration scheme [14].

In the next section, the eigenvalues of the Jacobian matrix are calculated numerically, and through this method, the influence of the forward speed, grid irregularities and the choice of boundary conditions can be investigated. 


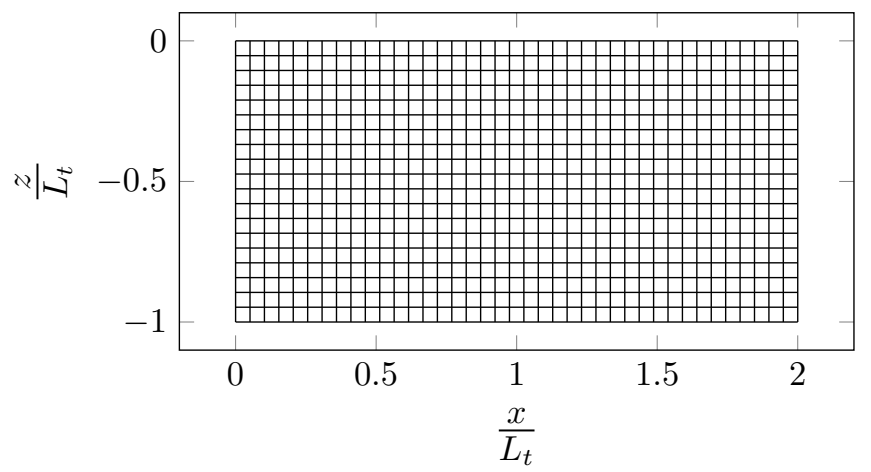

Figure 4: The uniform grid.
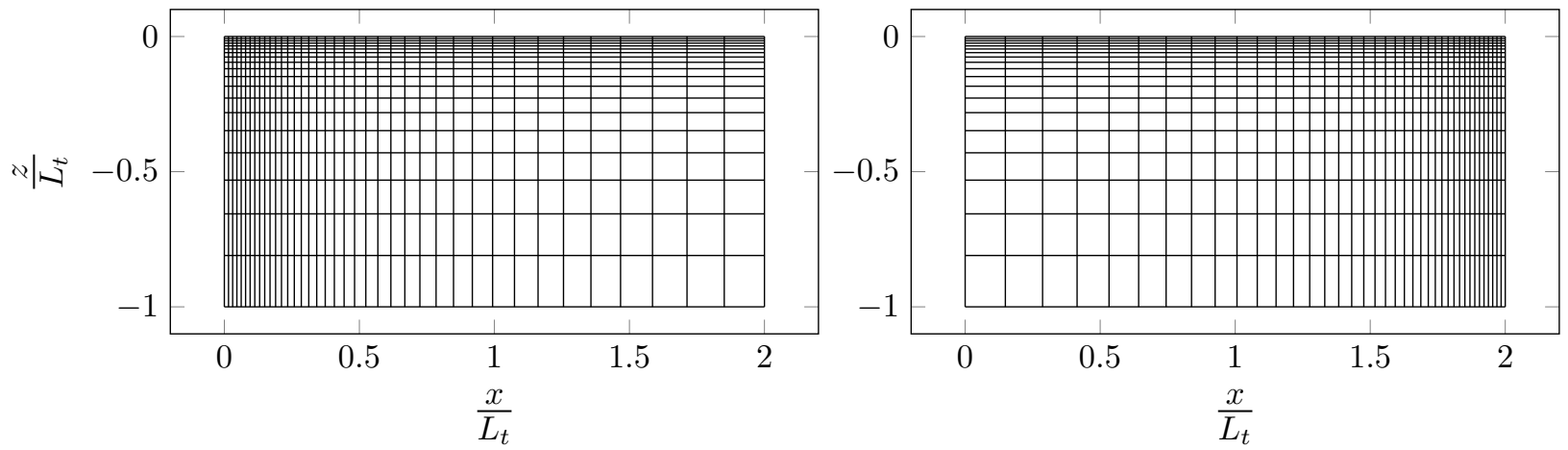

Figure 5: The stretched grid shown in the physical domain. The grid on the left is stretched towards the upwind boundary, and the grid in the right is stretched towards the downwind boundary. Both grids are also stretched in the $z$ direction.

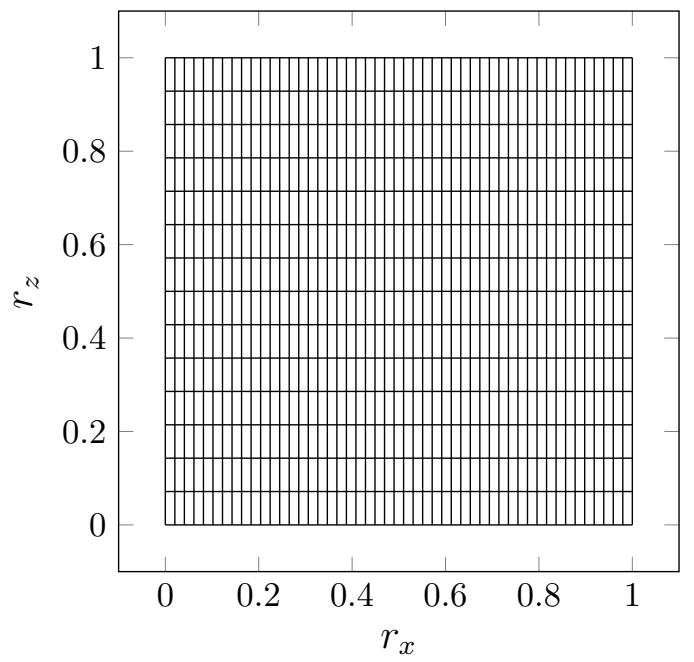

Figure 6: The stretched grid in the mapped domain.

\subsection{Stability analysis for representative grids}

The stability analysis is performed using the grids shown in Figures 4 to 6 . The first case in Figure 4 is a uniform spacing grid in the physical domain. Figure 5 shows two strongly stretched grids in the physical domain. Note that in this paper stretching towards a specific direction always is equivalent to the grid spacing being increases along that direction. The corresponding uniform computational grid for the stretched grids after applying a grid transformation is shown in Figures 6 . The characteristic 

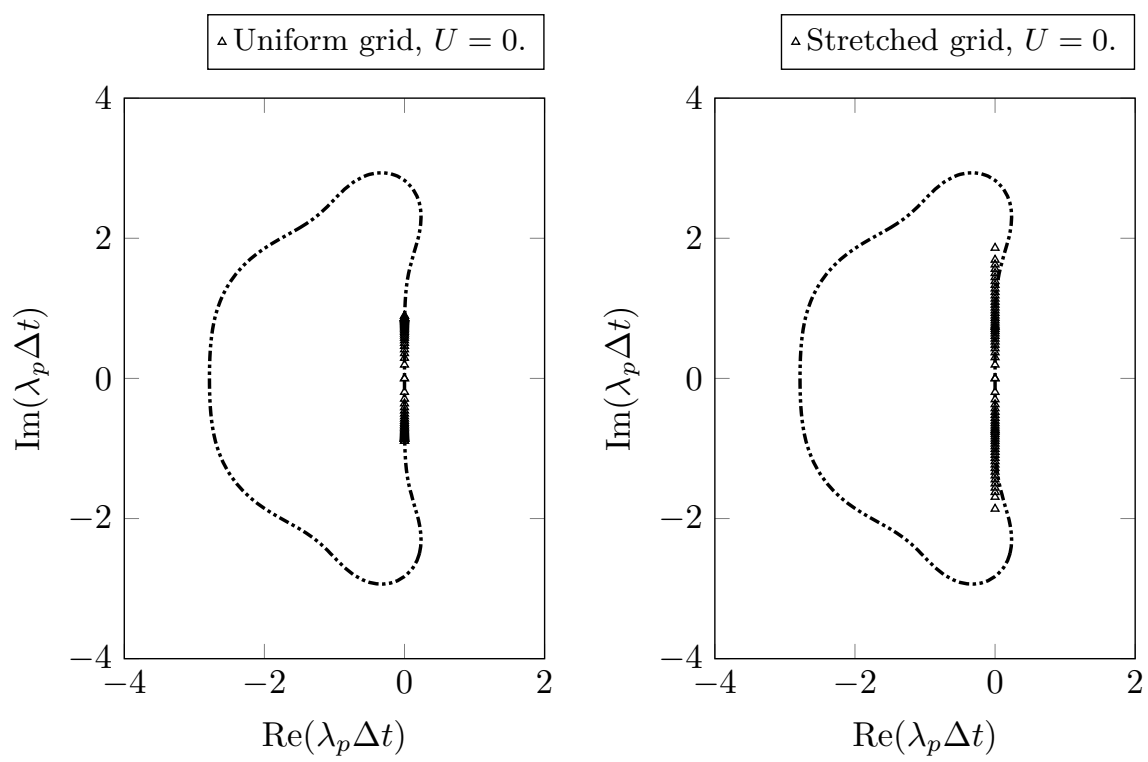

Figure 7: Eigenvalues for the uniform and the stretched grid when $U=0$.

length $L_{t}$ is chosen to be the depth of the domain. The grid stretching is defined by the following coordinate transformation [23]

$$
\begin{aligned}
& r=B+\frac{1}{\tau} \sinh ^{-1}\left[\left(\frac{x}{x_{c}}-1\right) \sinh (\tau B)\right], \\
& x=x_{c}\left\{1+\frac{\sinh [\tau(r-B)]}{\sinh (\tau B)}\right\},
\end{aligned}
$$

where the $x$ is the physical coordinate and $r \in[0,1]$ is the computational coordinate. The parameter $B$ is given by

$$
B=\frac{1}{2 \tau} \ln \left[\frac{1+\left(e^{\tau}-1\right)\left(x_{c} / L_{t}\right)}{1+\left(e^{-\tau}-1\right)\left(x_{c} / L_{t}\right)}\right] \quad 0<\tau<\infty,
$$

where $h$ is the length of the physical domain and $x_{c}$ is the point towards which the grid points are clustered. The strength of the stretching is controlled by the parameter $\tau$. The metric of the transformation is then:

$$
\frac{\partial r}{\partial x}=\frac{\sinh (\tau B)}{\tau x_{c} \sqrt{1+\left[\left(x / x_{c}\right)-1\right]^{2} \sinh ^{2}(\tau B)}} .
$$

\subsubsection{The $U=0$ case (Uniform and non-uniform grid)}

For $U=0$, and using centered schemes, the eigenvalues of the Jacobian matrix are all purely imaginary as shown in Figure 7 both for the uniform and the stretched grid. Here the dot-dashed line shows the stability region of the Runge-Kutta time integration scheme. The requirement for stability in this case is that $\Delta t \lambda_{p} \lesssim 2.7$, the point where the stability region crosses the imaginary axis.

\subsubsection{The $U \neq 0$ case (Uniform grid with a centered stencil)}

In this section we investigate the influence of the boundary treatment on the numerical stability of the scheme. We consider the uniform grid case with a forward speed corresponding to the Froude number $F_{n}=U / \sqrt{g L_{t}}=0.226$. Under these conditions, information is convected from right-to-left so that points to the left are downwind of those to the right. The following 3 sets of the boundary conditions are considered to perform the stability analysis: 

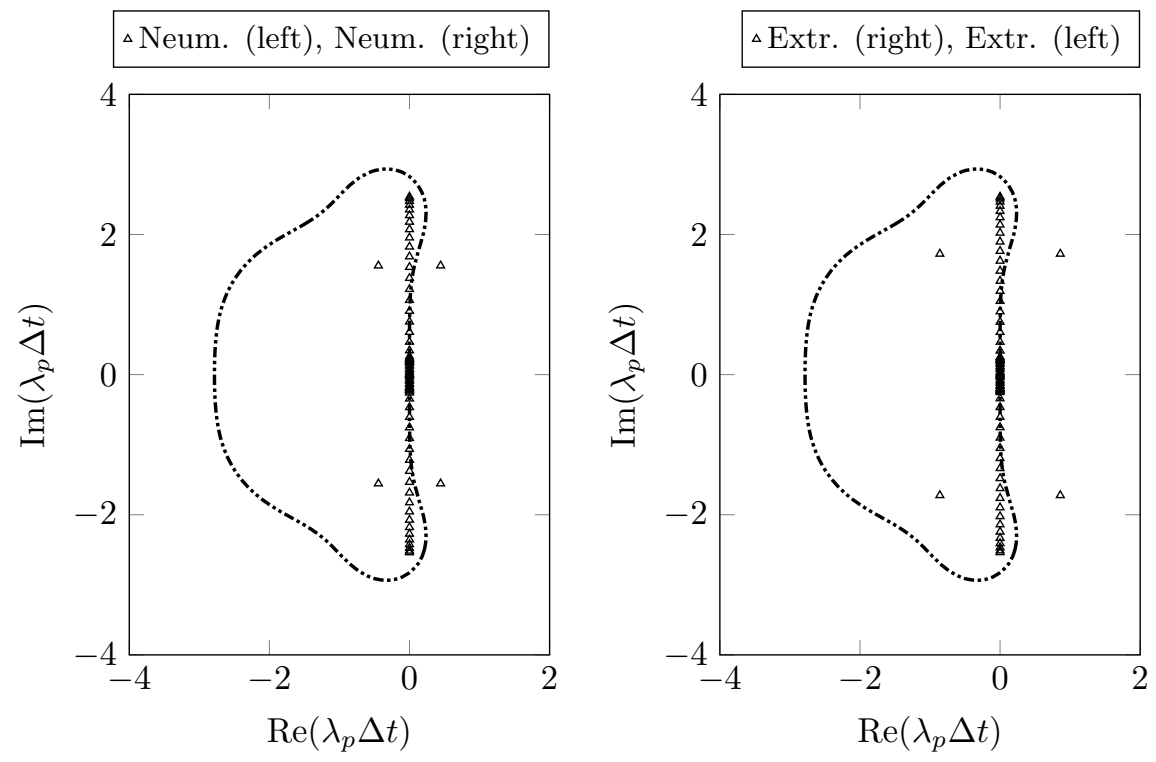

Figure 8: Eigenvalues for the uniform grid with the centered stencil when $U \neq 0$. The plot in the left is showing the case where the Neumann condition is applied both at the inflow and the outflow boundaries. The plot in the right is showing the case when the extrapolation is applied at both the inflow (right) and the outflow (left) boundaries.
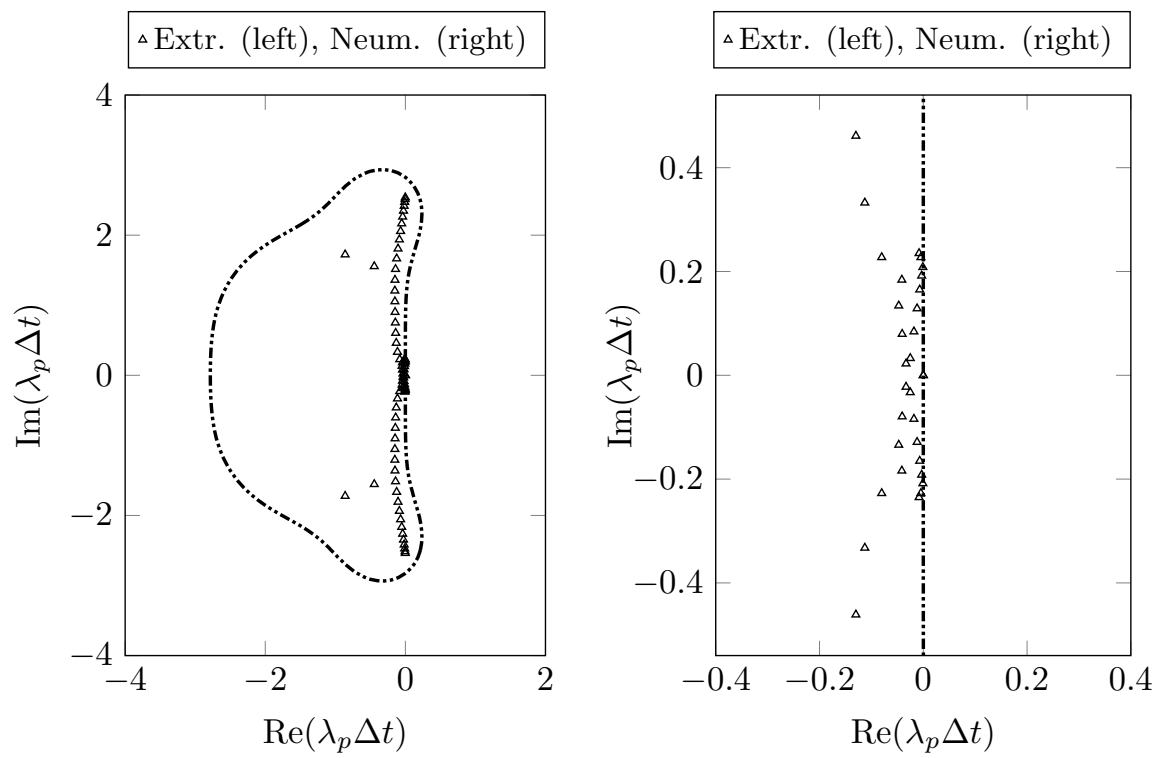

Figure 9: Eigenvalues for the uniform grid with the centered stencil when $U \neq 0$. The extrapolation condition is used at the left boundary (outflow) and the Neumann condition is applied at the right boundary (inflow) at the free surface. Expanded view near the origin is also shown in the right plot. 


\section{Neumann condition both at the left and at the right boundary}

The eigenvalues for this case are presented in Figure 8 (left). As can be seen, two positive real eigenvalues appear indicating an unconditionally unstable solution. This is however expected, as the solution at the left boundary (outflow) should be determined by the solution from the neighboring points from the upwind side of the domain. Applying the Neumann condition at this boundary acts as a sort of over-specification the solution, which results in reflections and the subsequent unstable solution. This situation is similar to the case of the one-dimensional advection equation with a Neumann condition at the outflow boundary [14]. Later, in the 3rd case it is shown how a stable solution can be obtained by replacing the Neumann condition at the left side with the extrapolation condition. The extrapolation at this side sounds a logical choice, as it is not specifying any new condition at that boundary and just uses the information from inside the computational domain to set the ghost points.

\section{Extrapolation both at the right and at the left boundary}

Figure 8 (right) shows the eigenvalues when extrapolation is applied both at the right and the left boundaries on the free surface. The positive real eigenvalues here again indicate an unconditionally unstable scheme. This is caused by an effective down-winding of the convective derivative at the up-stream boundary when extrapolation is used there. To illustrate this, consider the right-hand boundary grid point $i=N_{x}$ where the derivative (using a second-order scheme) is given by:

$$
\phi_{i}^{(1)}=\frac{1}{2 \Delta x}\left(\phi_{i+1}-\phi_{i-1}\right),
$$

with $\Delta x$ the grid spacing and $\phi_{i}^{(1)}$ denoting the $x$-derivative at grid point $i$. If the ghost point value $\left(\phi_{i+1}\right)$ is now extrapolated (to second-order) from the physical points, it will be given by:

$$
\phi_{i+1}=3 \phi_{i}-3 \phi_{i-1}+\phi_{i-2} .
$$

Inserting this into (4.14) gives:

$$
\phi_{i}^{(1)}=\frac{1}{2 \Delta x}\left(3 \phi_{i}-4 \phi_{i-1}+\phi_{i-2}\right),
$$

which is exactly the 3-point, second-order, one-sided derivative scheme obtained by using only the physical domain points. Thus, extrapolation at the upwind boundary is in fact equivalent to downwinding the derivative operator, which is always unstable.

\section{Extrapolation at the left and Neumann condition at the right boundary}

Based on the explanations from the previous two cases, the required set of boundary conditions for a stable numerical scheme is in fact extrapolation at the left boundary and the Neumann condition at the right boundary. The eigenvalues for this situation are shown in Figure 9, which indicates a stable scheme. This set of boundary conditions is in fact the reason for specifying the outflow and inflow regions at the free surface in Figure 2 to apply the Neumann and the extrapolation condition.

Note that from now on this set of boundary conditions is used for all other stability analyses performed in the following sections.

\subsubsection{The $U \neq 0$ case (Non-uniform grid with a centered stencil)}

Grid irregularities and grid stretching can also produce instability. In Appendix A, we present a von Neumann stability analysis to show this for the simple one-dimensional advection equation discretized on a stretched grid using the centered stencil. In this section this fact is illustrated using the same matrix-based stability analysis for the stretched grids shown in Figure 5. The analysis is performed both in the physical domain and in the mapped domain. 

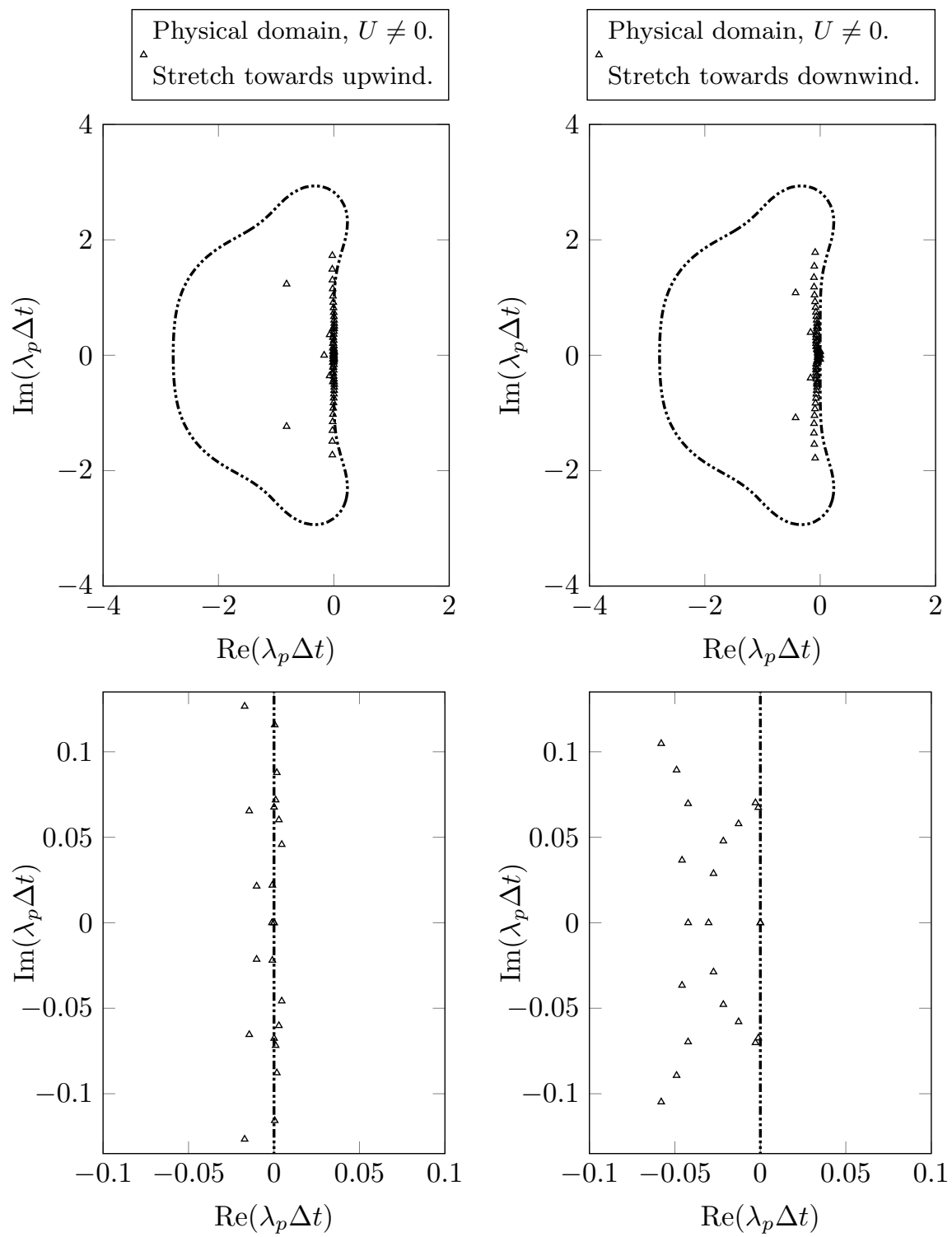

Figure 10: The influence of the grid stretching on the numerical stability when $U \neq 0$. The computation is performed in the physical domain and the convective derivatives are calculated using the centered stencil. In the left plot the grid is stretched towards the upwind boundary, and in the right plot the grid is stretched towards the downwind boundary. Expanded views near the origin are shown in the bottom two plots. 


\section{Physical domain}

First the grid in Figure 5 (left) is considered. The $\mathbf{D} \mathbf{z}^{\mathbf{0}}$, and $\mathbf{D}_{\mathbf{x}}$ matrices are calculated based on the discretization in the physical domain and for $F_{n}=0.226$, and the results are shown in Figure 10 (left). In the physical domain, the grid points which are located at the downwind side (towards the cluster point) have smaller spacings than those toward the upwind side, and will therefore be weighted more heavily by the finite-difference coefficients. This suggests a down winding effect, which intuitively might be expected to produce instability. Figure 10 (left) indeed shows that some eigenvalues have positive real parts indicating instability. Exactly the opposite behavior can be seen if the stretching is performed towards the upwind boundary (the grid shown in Figure 5 (right)). The results for this case are shown in Figure 10 (right).

\section{Mapped domain}

For a 2D domain, the convective derivative is computed in the mapped domain from:

$$
\frac{\partial \phi}{\partial x}=\frac{\partial r}{\partial x} \frac{\partial \phi}{\partial r}
$$

In this case, the finite-difference weights for $\partial \phi / \partial r$ are calculated on the uniform computational grid, so that each row in the $\mathbf{D}_{\mathbf{x}}$ matrix is symmetric around the center point of the stencil. Each row is then scaled by the local grid stretching factor $\partial r / \partial x$, which does not break the symmetry. The stability eigenvalues, with the convective derivatives computed in the mapped domain, are shown in Figures 11. Note that the order of the results in the figure corresponds to the grids shown in Figure 5. In contrast to the case of the physical domain, grid stretching towards the upwind boundary Figure 5(left) leads to stable solutions, as can be seen from Figure 11 (left). Moreover the grid stretching towards the downwind boundary would cause instability as is indicated by some positive and real eigenvalues in Figure 11 (right). This behavior has also been observed in the numerical simulations which are based on the computation in the mapped domain. As is shown in the next section, the biased stencil will in these cases provide the required stability. 

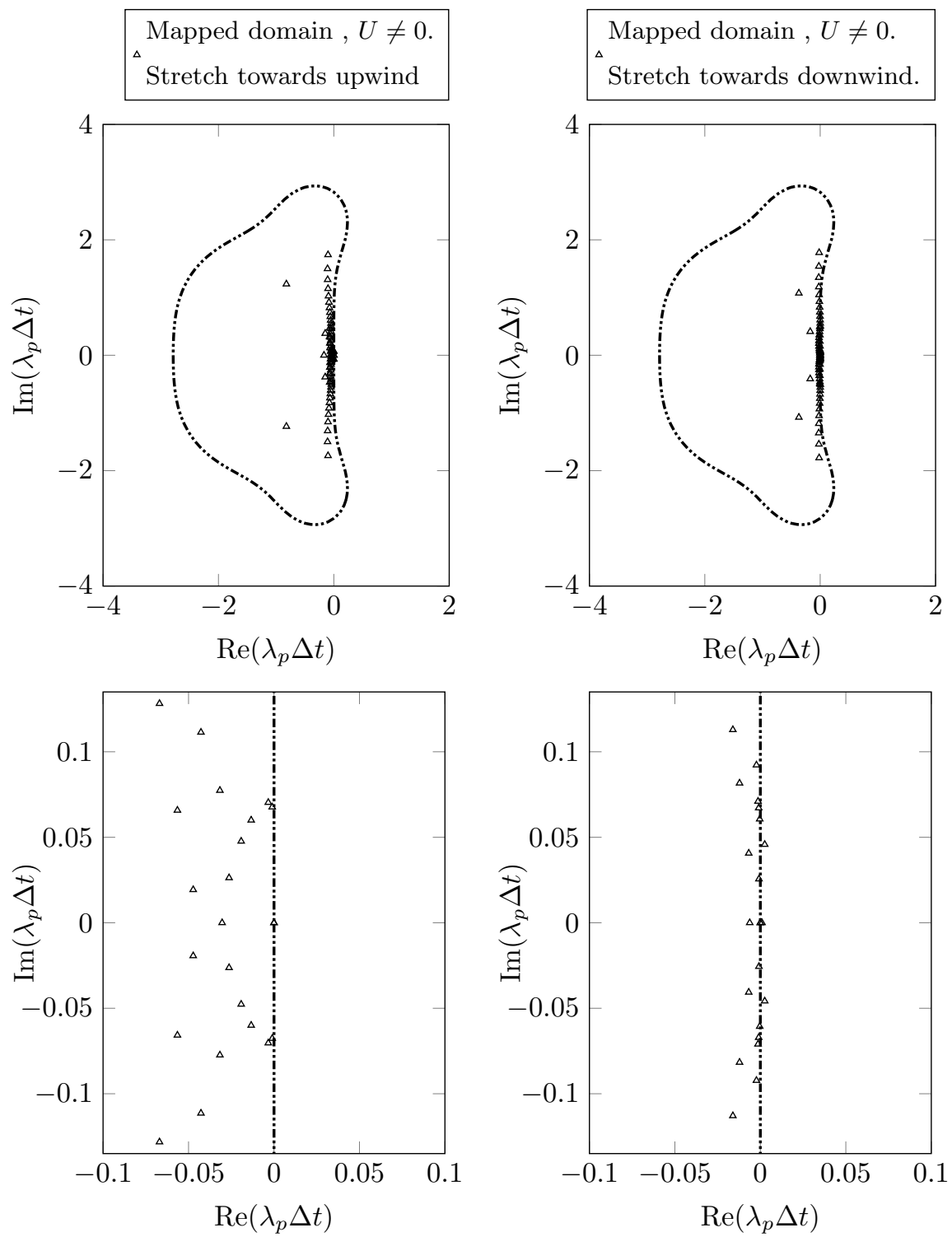

Figure 11: The influence of the grid stretching on the numerical stability when $U \neq 0$. The computation is performed in the mapped domain and the convective derivatives are calculated using the centered stencil. In the left plot the grid is stretched towards the upwind boundary, and in the right plot the grid is stretched towards the downwind boundary. Expanded views near the origin are shown in the bottom two plots. 


\subsubsection{The $U \neq 0$ case (Non-uniform grid with upwind biased stencil)}

In order to ensure stability in the case of grid stretching and/or irregularities, an upwind biased stencil can be used for the calculation of the convective derivatives. The two up-winded schemes considered here are shown in Figure 3. This choice seems logical in view of the insight obtained regarding the influence of the relative size of the downwind side coefficients on the stability. The stability eigenvalues for the one-point up-winded scheme, denoted by $(2-3)$, are shown in Figure 12. Note that the results are for both grids shown in Figure 5. The similar influence is observed if the discretization is performed in the mapped domain that is expected according to the argument given in the previous section. The negative real eigenvalues in this case are a sign of numerical diffusion which in fact provides a sort of stability to the scheme. The use of one more biased points towards the upwind boundary as shown in Figure 3 (bottom) would also leads to the same behavior in the numerical scheme. Note that in all computations presented in the next sections the convective derivatives at the free-surface conditions are calculated using the $(2-4)$ stencil. 

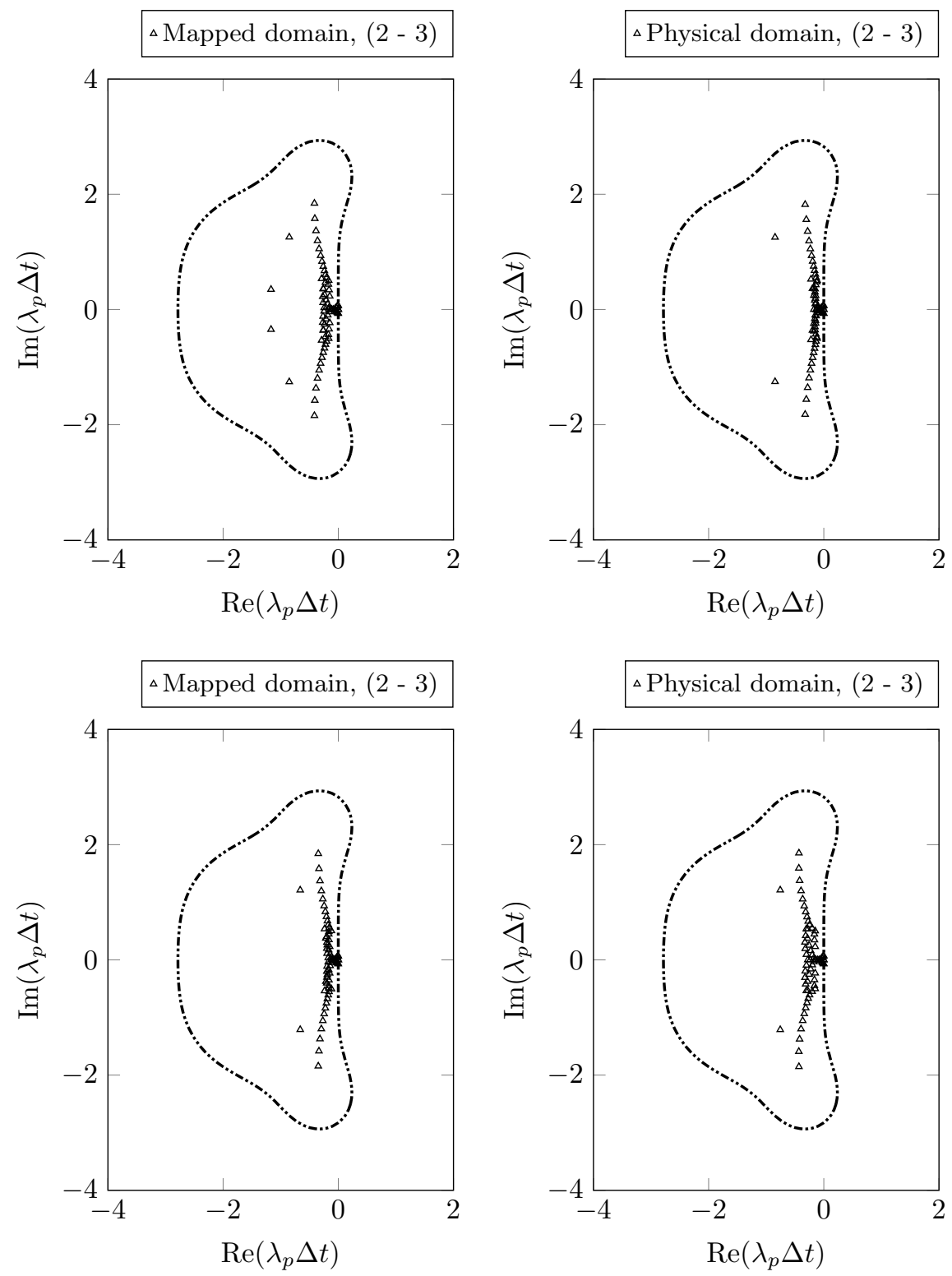

Figure 12: Eigenvalues in the case of the non-uniform grid with $U \neq 0$. The convective derivatives are calculated using the upwind-biased stencil $(2-3)$. The top and bottom plots are for the grids shown in the left and the right side of Figure 5 respectively. 


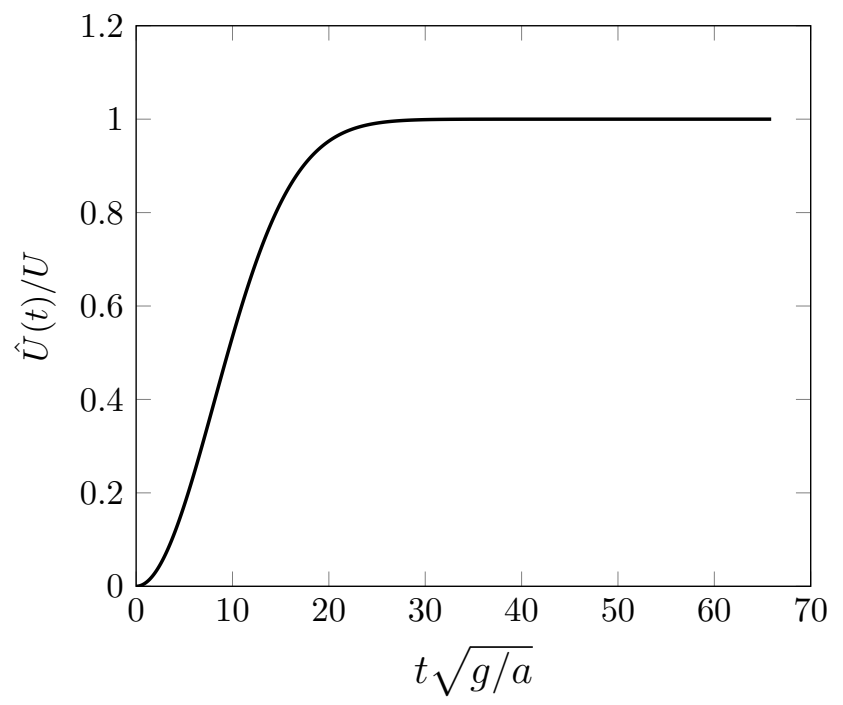

Figure 13: The ramp function for the body boundary condition.

\section{Applications to the steady wave resistance problem}

The developed numerical tool whose implementation methodology has been explained in the previous sections, is used here to calculate the wave resistance force for the case of a submerged sphere and the Wigley hull. The developed solver is named OceanWave3D-Seakeeping. Note that a convergence study for this solver has been performed in [1] using 2 test solutions which are the double-body potential for a sphere and the monochromatic wave on a periodic domain.

In the first place, and just in order to illustrate the stability of the numerical scheme, a simple twodimensional case is studied where a semi-infinite floating cylinder moves in otherwise calm water. In all numerical calculations which are presented in this section the convective derivatives in the free-surface boundary conditions are calculated using the biased stencil differencing scheme. In this developed numerical tool for the wave resistance problems, the body boundary condition (2.4) is applied in time domain using a ramp function given as:

$$
\frac{\hat{U}(t)}{U}=1-\exp \left[-0.15(t \sqrt{g / a})^{2}\right],
$$

where the characteristic length of the body is given by $a$. See also Figure 13.

\subsection{Floating circular cylinder}

In this case the overlapping grid is generated for the semi-infinite floating cylinder with radius $a$. See Figure 14. A numerical simulation is carried out where the the body is moving in the positive $x$ direction with a Froude number of $F_{n}=U / \sqrt{g a}=0.6$. According to [24] a wave train of the length $\lambda / a=2 \pi F_{n}^{2}=2.26$ is generated behind the body. See Figure 15. Note that the free-surface elevation is denoted by $\zeta$ and the length along the tank is shown by $L_{t}$.

Based on the definition of the inflow and outflow regions shown in Figure 2, there are two inflow regions marked by $N_{1}$ and $N_{2}$. The homogeneous Neumann condition is applied for the free-surface elevation $\zeta$ at the points $N_{1}$ and $N_{2}$ to calculate the values at the ghost points, whereas for the velocity potential $\phi$, the relevant non-homogeneous Neumann conditions are used at $N_{1}$ and $N_{2}$ for setting the ghost points. Moreover at two outflow region marked by $E_{1}$ and $E_{2}$ in Figure 15, the ghost points for both the velocity potential and the surface elevation are set using the extrapolation from inside the domain. 


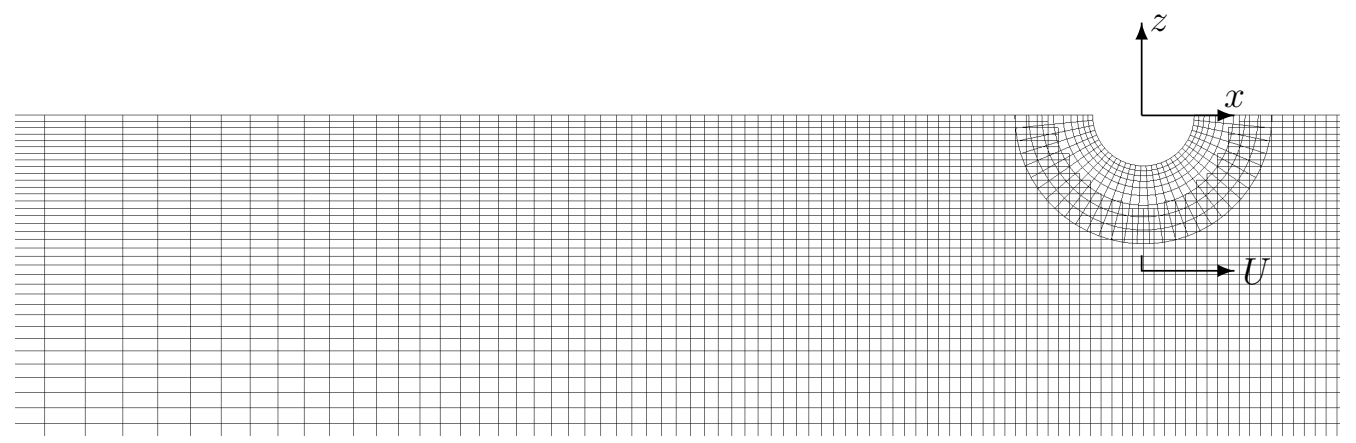

Figure 14: The 2D overlapping grid for the floating cylinder.

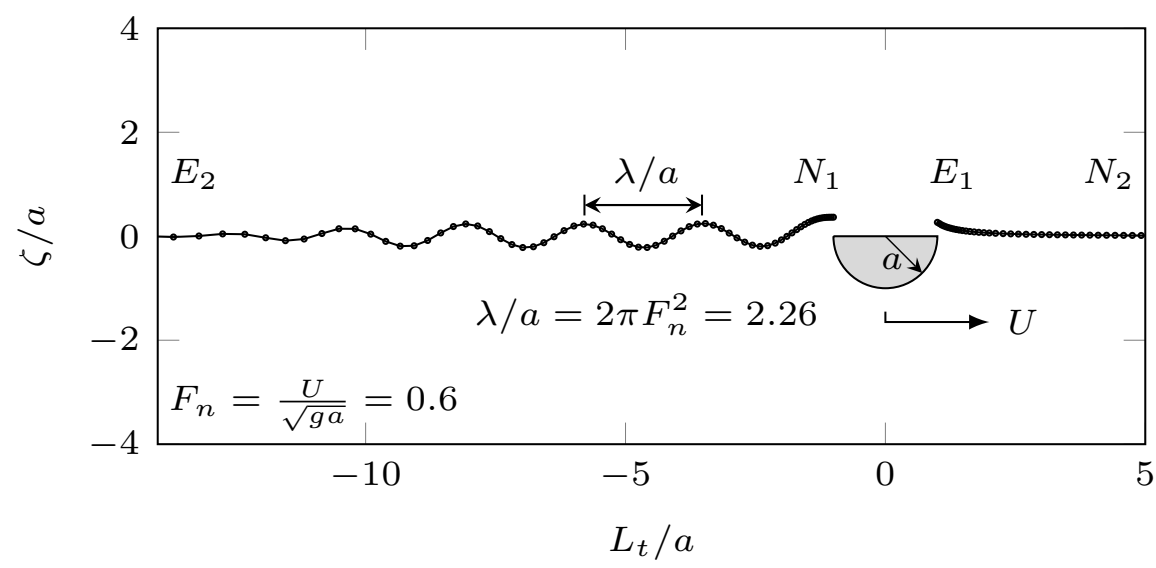

Figure 15: The floating cylinder in the steady forward speed $F_{n}=0.60$. 


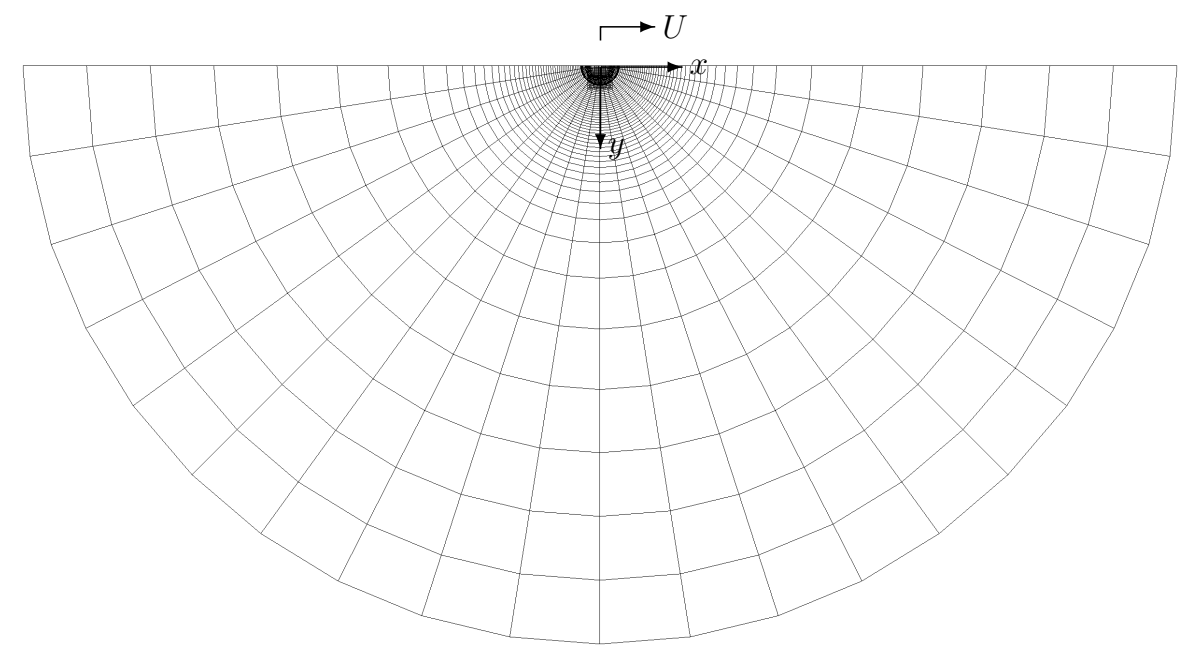

Figure 16: Part of the 3D overlapping grid for the submerged sphere (top view).

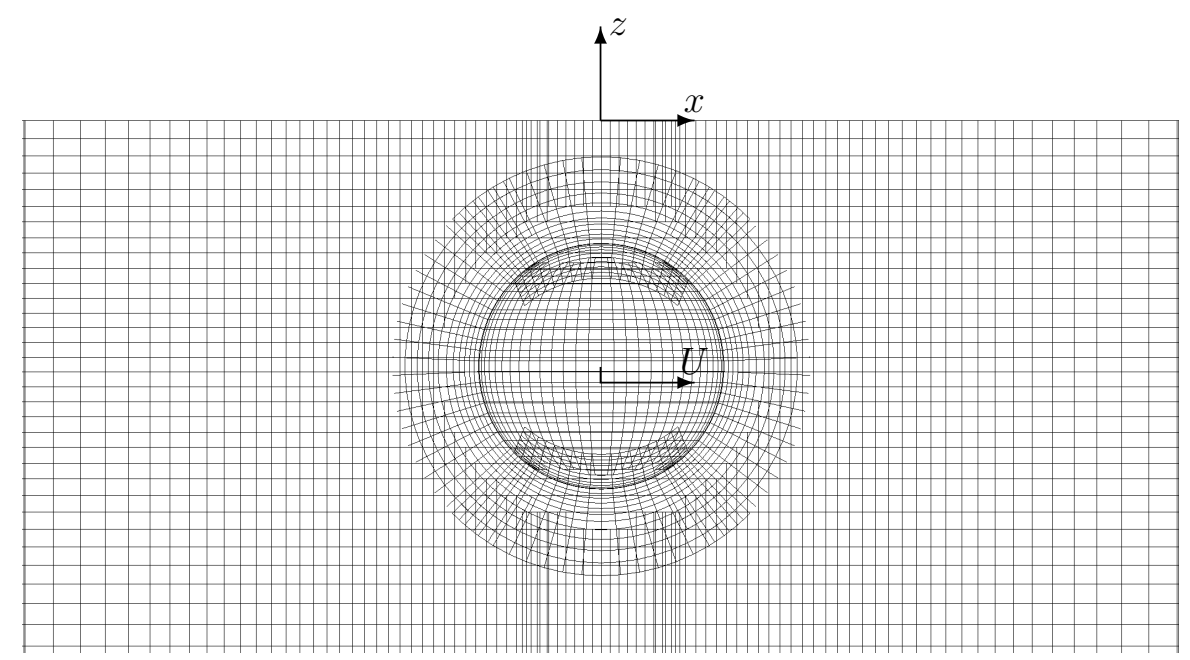

Figure 17: Part of the 3D overlapping grid for the submerged sphere (side view).

\subsection{Submerged sphere}

An analytical solution exists for the wave resistance problem of a submerged sphere, and is given by [25]. In this work a submerged sphere with radius $a$, and the submergence depth of $h=2.0 a$ moves with different Froude numbers $\left(F_{n}=U / \sqrt{g h}\right)$ in an open water basin. Part of the overlapping grid to be used for the numerical calculation of this submerged sphere is shown in Figure 16 and 17. The wave resistance force is calculated by integrating the following pressure force around the surface of the body:

$$
p=-\rho\left[\frac{\partial \phi}{\partial t}-U \frac{\partial \phi}{\partial x}+\frac{1}{2} \nabla \phi \cdot \nabla \phi\right] .
$$

Note that in comparison with the linearized force in equation (2.7), the second order term $\nabla \phi \cdot \nabla \phi$, has been added here in order to be consistent with the analytical solution in [25]. The comparison with the analytical solution is given in Figure 18. Note that the results are for the Froude numbers in the range $0.5,0.6, \cdots, 1.1$. 

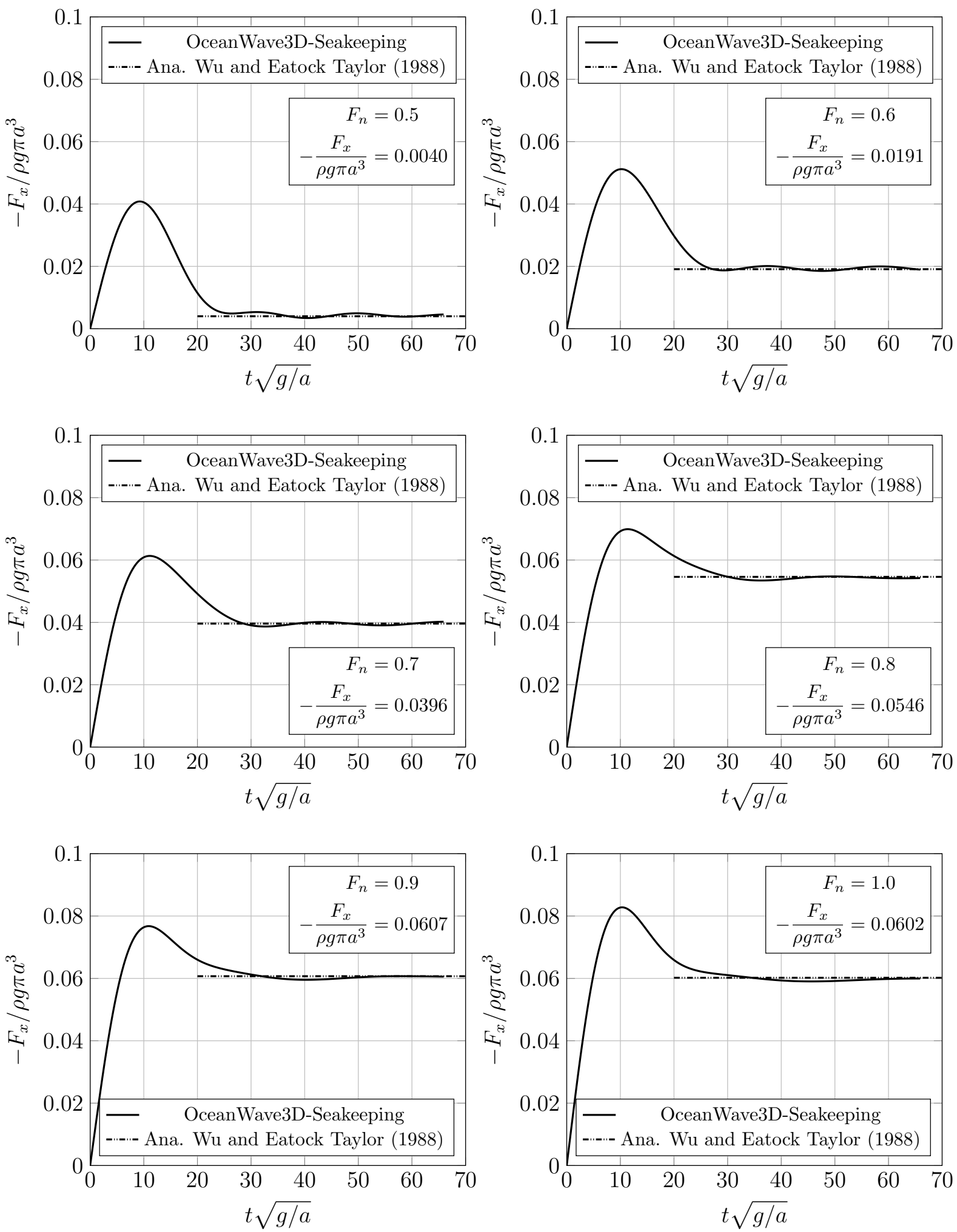

Figure 18: Wave resistance force for the submerged sphere for a range of Froude numbers. 


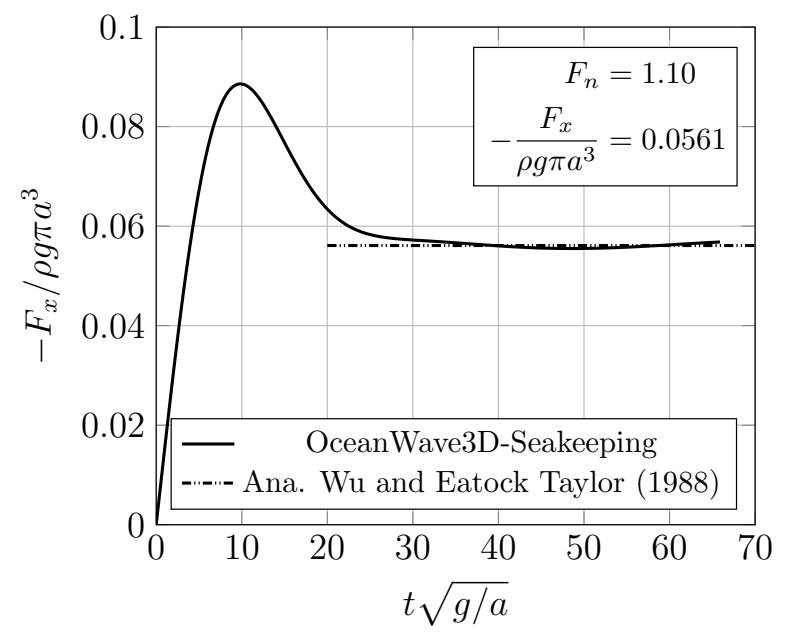

Figure 18 (cont.): Wave resistance force for the submerged sphere for a range of Froude numbers.
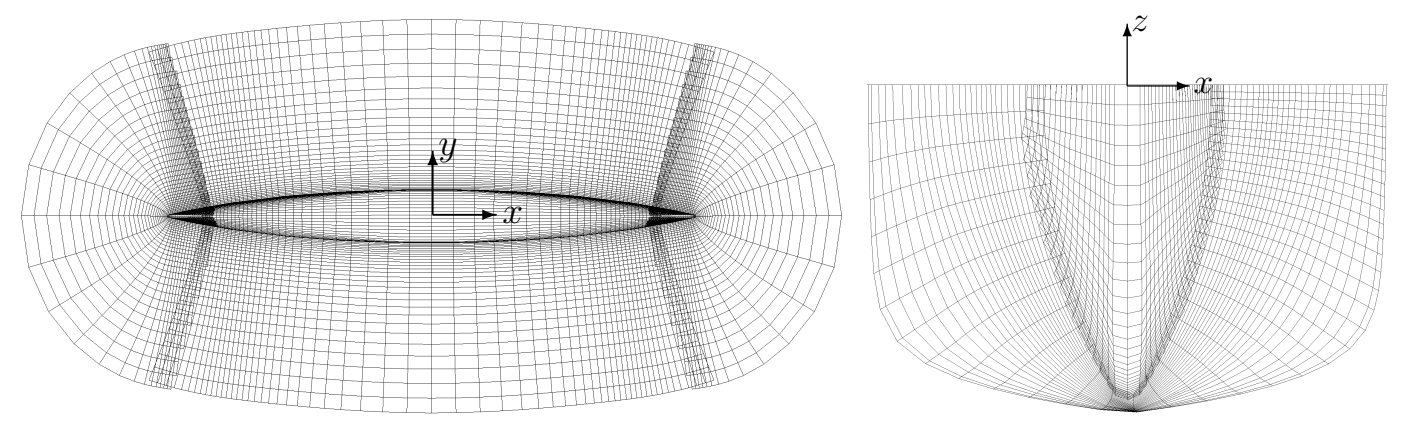

Figure 19: Part of the final overlapping grid for the Wigley hull.

\subsection{Wigley hull}

\subsubsection{Grid generation}

In the case of the cylinder and the sphere, the grid generation process is rather straightforward, as in Ogen [26] the relevant analytical mappings are utilized to define the required transformations. For an arbitrary geometry like the ship hull, however, the more general hyperbolic grid generation technique [16] is used in Ogen, where the grid generation is based on a CAD definition of the surface geometry. A number of component grids can be generated to cover the ship geometry, and a cylindrical volume grid is also generated to act as the background grid for the basin. The final overset grid is then obtained by combining all these component grids, see also Figure 19. More details on grid generation using this method for ship geometries is given in [1].

\subsubsection{Wave resistance}

The calculation for the wave resistance of the Wigley hull (type I) has been performed for the generated overset grid from the previous section. The wave resistance force $F_{x}$ is calculated by integration of the pressure force from equation (5.2) over the surface of the ship hull. As mentioned earlier the simulation is continued until a steady state force is obtained. According to [27] in the wave resistance problem the time-domain force oscillates asymptotically around the steady resistance force $a_{3}$ as follows:

$$
F_{x}^{\infty}=\frac{1}{t}\left[a_{1} \sin \left(\omega_{c} t\right)+a_{2} \cos \left(\omega_{c} t\right)\right]+a_{3},
$$



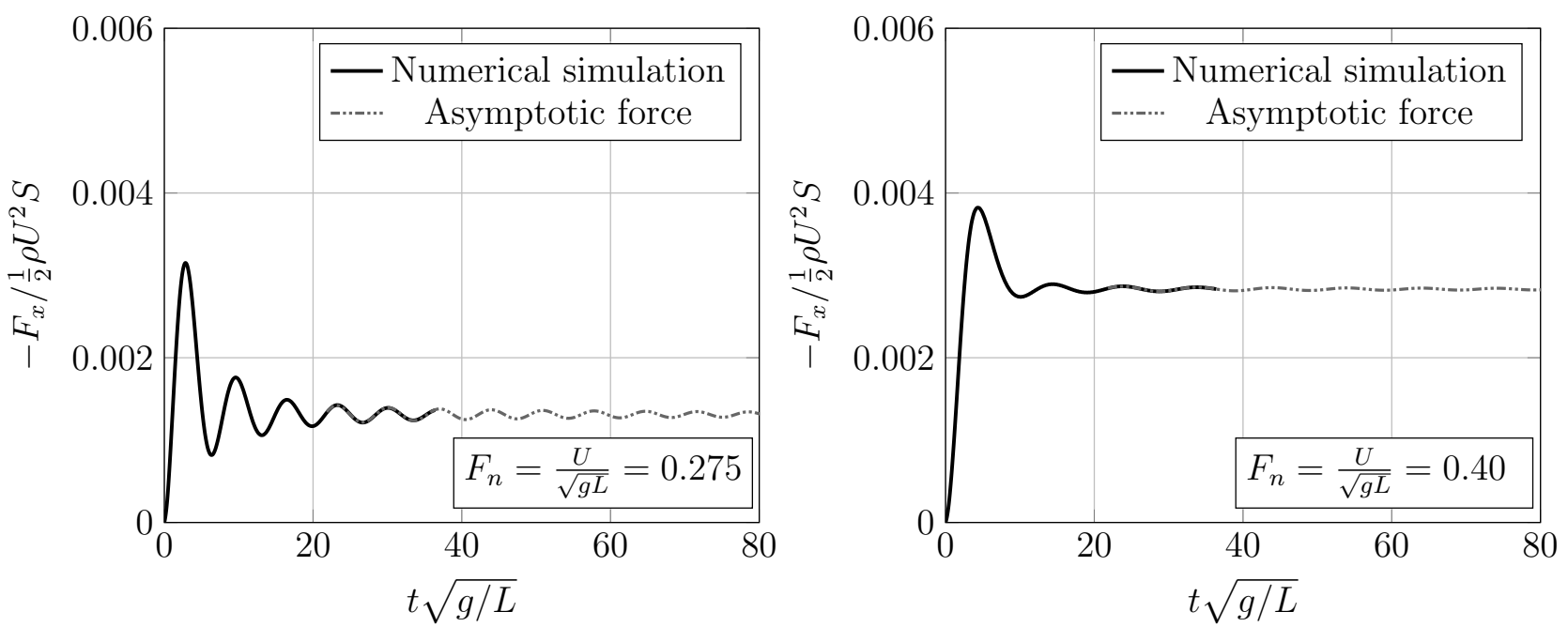

Figure 20: Asymptotic continuation of the resistance force for the Wigley hull.

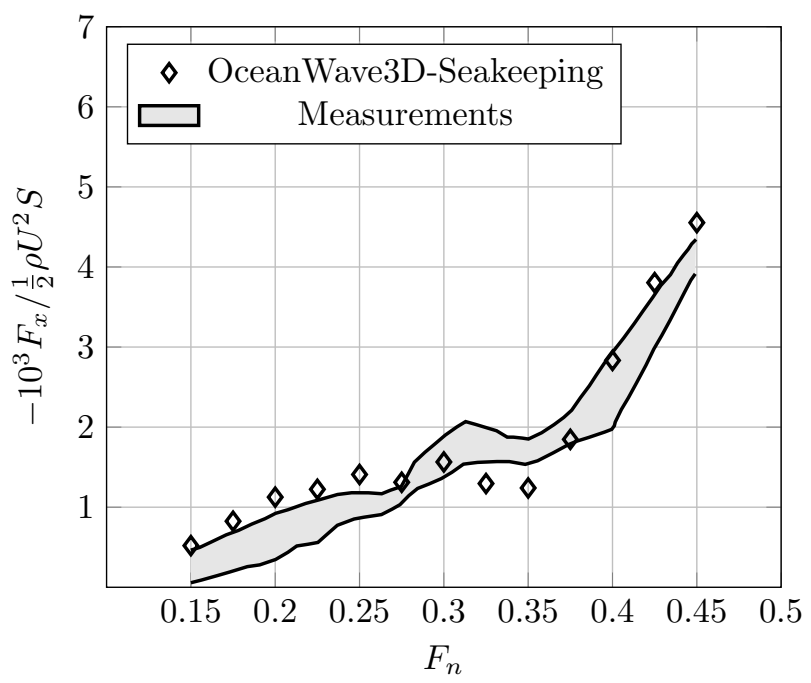

Figure 21: Wave resistance force for the Wigley hull.

where the critical frequency is defined as:

$$
\omega_{c}=\frac{g}{4 U}
$$

The coefficients $a_{1}, a_{2}$ and $a_{3}$ can be obtained by the least-square fitting of asymptotic relation (5.3) to a fraction of the time-domain force which is obtained by the numerical simulation. The results of the least-square fitting over the resistance force for two Froude numbers $U / \sqrt{g L}$ are shown in Figure 20. Note that $L$ is the ship length and the wave resistance is non-dimensionalized by $\frac{1}{2} \rho U^{2} S$, where $S$ is the wetted surface of the ship hull.

The wave resistance force, i.e $a_{3}$, has been calculated based on the described procedure for the Froude numbers $0.15,0.175, \cdots, 0.425,0.45$. The results are compared against the scatter of different experiments [28] performed for the Wigley hull, and are shown in Figure 21. For the case of Froude number 0.45 , the non-dimensional free-surface wave elevation $\frac{\zeta}{L}$ has been also plotted in Figure 22 . 


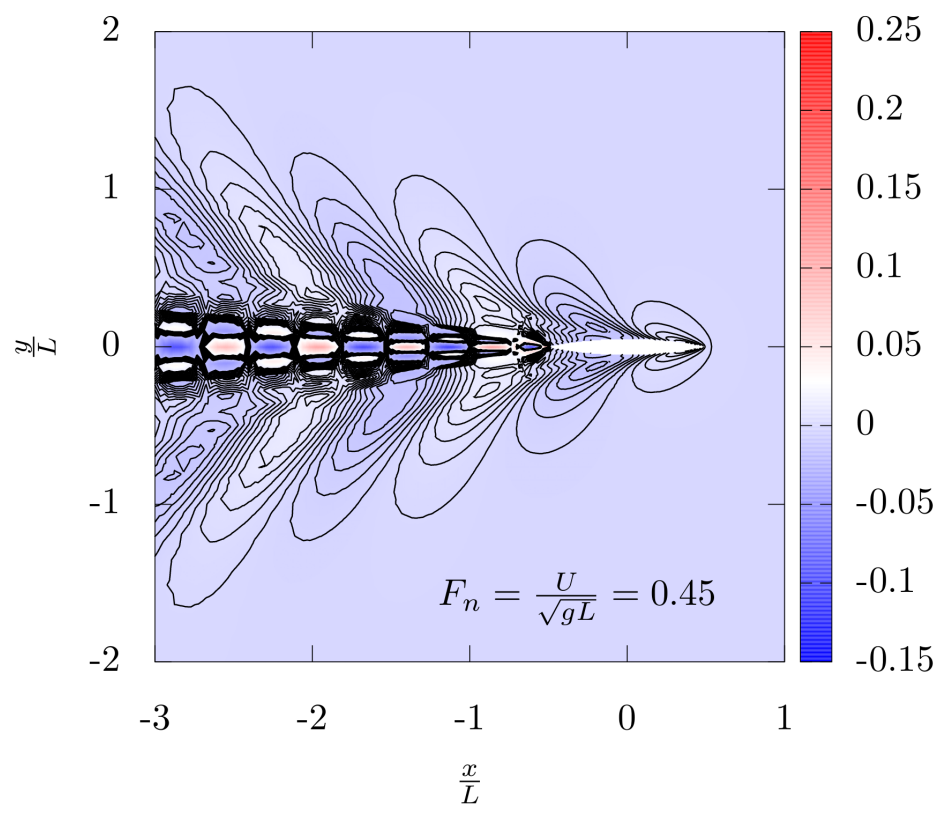

Figure 22: Surface wave elevation $\left(\frac{\zeta}{L}\right)$ for the Wigley hull, $F_{n}=0.45$.

\section{Conclusions}

A matrix-based stability analysis has been used to investigate the numerical stability of the NeumannKelvin formulation of the wave resistance hydrodynamic problem. The influence of the boundary conditions on the numerical stability has been demonstrated. It is shown that with a proper choice of the Neumann condition for $\phi$ and $\zeta$ in the inflow region $(\boldsymbol{n} \cdot \boldsymbol{W}>0)$ at the boundaries, one can avoid using downwind extrapolation and the resultant instability. Moreover in the outflow region $(\boldsymbol{n} \cdot \boldsymbol{W}<0)$, extrapolation can be applied safely from the upwind direction. This will prevent the rise of the spurious waves and numerical instability, which is due to an over-specification of the boundary condition in the outflow region. The stability analysis also shows how the centered scheme sometimes becomes effectively down-winded on stretched grids. In the physical domain this behavior is intuitively clear from the weighting of the finite-difference coefficients which emphasize the closer points. Although not intuitively obvious, the analysis also shows that grid stretching results in instability when the convective operator is developed in a uniform mapped domain. Two strategies are proposed to achieve stability: upwind-biased stencils; and centered stencils in combination with a mild least-squares filter. The application of this numerical framework is shown in the solution for the wave resistance of a submerged sphere and the Wigley hull. Numerical results for the wave resistance of the submerged sphere compare very well with the analytical solution. Additionally good agreement has been observed between the experimental measurements and the numerical solution for the wave resistance of the Wigley hull. The developed stable numerical method in this paper will be employed next to solve other forward-speed hydrodynamic problems. These will be included in a seakeeping tool for calculation of wave loading and response of sailing ships in the ocean.

\section{Acknowledgement}

The financial support provided by The Orients Fund and The Danish Maritime Fund is highly appreciated. The helpful discussions on the numerical stability with Assoc. Prof Allan Peter Engsig-Karup 
and Dr. Ole Lindberg both at the Technical University of Denmark, are also deeply acknowledged. 


\section{References}

[1] M. Amini-Afshar and H. B. Bingham, "Solving the linearized forward-speed radiation problem using a high-order finite difference method on overlapping grids," Applied Ocean Research, vol. 69, pp. 220-244, 2017.

[2] M. Amini-Afshar and H. B. Bingham, "Pseudo-impulsive solutions of the forward-speed diffraction problem using a high-order finite-difference method," Applied Ocean Research, vol. 80, pp. 197$219,2018$.

[3] L. Larsson, F. Stern, and M. Visonneau, "Numerical ship hydrodynamics," in Gothenburg 2010 a Workshop on Numerical Ship Hydrodynamics, Gothenburg, pp. 1-32, Springer, 2010.

[4] H. Raven, A. Van der Ploeg, A. Starke, and L. Eça, "Towards a CFD-based prediction of ship performanceprogress in predicting full-scale resistance and scale effects," Proceedings of RINACFD-2008, London, UK, 2008.

[5] F. Stern, J. Yang, Z. Wang, H. Sadat-Hosseini, M. Mousaviraad, S. Bhushan, and T. Xing, "Computational ship hydrodynamics: Nowadays and way forward," International Shipbuilding Progress, vol. 60, no. 1-4, pp. 3-105, 2013.

[6] D. Nakos, Ship wave patterns and motions by a three dimensional Rankine panel method. PhD thesis, Massachusetts Institute of Technology, 1990.

[7] D. C. Kring, Time domain ship motions by a three-dimensional Rankine panel method. PhD thesis, Massachusetts Institute of Technology, 1994.

[8] Y. Kim, D. C. Kring, and P. D. Sclavounos, "Linear and nonlinear interactions of surface waves with bodies by a three-dimensional Rankine panel method," Applied Ocean Research, vol. 19, no. 5, pp. 235-249, 1997.

[9] T. Vada and D. Nakos, "Time-marching schemes for ship motion simulations," in Proceedings of the 8th International Workshop on Water Waves and Floating Bodies, St. Johns, Newfoundland, Canada, pp. 155-158, 1993.

[10] B. Büchmann, "Accuracy and stability of a set of free-surface time-domain boundary element models based on b-splines," International journal for numerical methods in fluids, vol. 33, no. 1, pp. $125-155,2000$.

[11] F. Kara and D. Vassalos, "Time domain computation of the wave-making resistance of ships," Journal of ship research, vol. 49, no. 2, pp. 144-158, 2005.

[12] H. B. Bingham and H. Zhang, "On the accuracy of finite-difference solutions for nonlinear water waves," Journal of Engineering Mathematics, vol. 58, no. 1-4, pp. 211-228, 2007.

[13] A. P. Engsig-Karup, H. B. Bingham, and O. Lindberg, "An efficient flexible-order model for 3d nonlinear water waves," Journal of computational physics, vol. 228, no. 6, pp. 2100-2118, 2009.

[14] R. J. LeVeque, Finite difference methods for ordinary and partial differential equations: steadystate and time-dependent problems, vol. 98. Siam, 2007.

[15] H. B. Bingham, M. Amini-Afshar, R. Read, and A. P. Engsig-Karup, "Stable finite difference discretizations of the forward speed seakeeping problem," in 29th International Workshop on Water Waves and Floating Bodies (IWWWFB), Osaka, Japan, 2014.

[16] W. M. Chan, "Hyperbolic methods for surface and field grid generation," in Handbook of Grid Generation (S. Thompson and Weatherill, eds.), ch. 5, pp. 5-1-5-26, CRC Press, 1998. 
[17] J. Newman, "The theory of ship motions," Advances in Applied Mechanics, vol. 18, pp. 221-283, 1979.

[18] J. W. Thomas, Numerical partial differential equations: finite difference methods, vol. 22. Springer, 1995.

[19] B. Fornberg, "Classroom note: Calculation of weights in finite difference formulas," SIAM review, vol. 40, no. 3, pp. 685-691, 1998.

[20] A. Savitzky and M. J. Golay, "Smoothing and differentiation of data by simplified least squares procedures.," Analytical chemistry, vol. 36, no. 8, pp. 1627-1639, 1964.

[21] J. Berland, C. Bogey, O. Marsden, and C. Bailly, "High-order, low dispersive and low dissipative explicit schemes for multiple-scale and boundary problems," Journal of Computational Physics, vol. 224, no. 2, pp. 637-662, 2007.

[22] M. Amini-Afshar, Towards Predicting the Added Resistance of Slow Ships in Waves. PhD thesis, DTU Mechanical Engineering, 2015.

[23] G. O. Roberts, "Computational meshes for boundary layer problems," in Proceedings of the Second International Conference on Numerical Methods in Fluid Dynamics, pp. 171-177, Springer, 1971.

[24] J. N. Newman, Marine Hydrodynam. The MIT press, 1977.

[25] G. Wu and R. E. Taylor, "Radiation and diffraction of water waves by a submerged sphere at forward speed," Proceedings of the Royal Society of London. A. Mathematical and Physical Sciences, vol. 417, no. 1853, pp. 433-461, 1988.

[26] W. D. Henshaw, "Ogen: An overlapping grid generator for overture," LANL unclassified report, pp. 96-3466, 1998.

[27] H. B. Bingham, Simulating ship motions in the time domain. PhD thesis, Massachusetts Institute of Technology, Department of Ocean Engineering, 1994.

[28] K. Bai and J. McCarthy, "Overview of results," in Proceedings of the Workshop on Ship Waveresistance Computations, David W. Taylor Naval Ship Research and Development Center, Maryland, USA, pp. 5-50, 1979. 


\section{A von Neumann stability analysis of the advection equation on a stretched grid}

In this appendix, a von Neumann stability analysis is presented for the one-dimensional (1D) advection equation discretized using a centered stencil on a stretched grid. This is meant to complement the matrix-based analysis presented above and illustrates the possible downwinding effect of grid stretching. The 1D, semi-discrete advection equation can be written:

$$
\frac{d f_{j}}{d t}=-\frac{1}{\Delta x} \sum_{k=1}^{r} c_{k} f_{j-a+k-1},
$$

where $f_{j}$ is the function value at position $x_{j}$ with $\Delta x$ a scaling to characterize the grid, $r$ is the total number of stencil points used with $a$ the number of points to the left, and $c_{k}$ is the finite difference coefficient of the scheme on stencil point $k$. Unit amplitude flow is assumed from left to right. Plugging in the Fourier mode:

$$
f(x, t)=a_{n}(t) e^{\mathrm{i} \frac{2 \pi}{n \Delta x} x},
$$

where $2 \leq n \leq \infty$, we get:

$$
\frac{\partial a_{n}}{\partial t}=-\frac{\lambda_{n}}{\Delta x} a_{n}
$$

Here

$$
\lambda_{n}=\sum_{k=1}^{r} c_{k} e^{\mathrm{i} \frac{2 \pi}{n} \xi_{k}},
$$

where $\xi_{k}=\left(x_{j}-x_{k}\right) / \Delta x$ is the relative stencil position and $-\lambda_{n} \Delta t / \Delta x$ is the stability eigenvalue of the scheme with $\Delta t / \Delta x$ the Courant number. We consider a 3 -point centered stencil for a grid which is stretched in the upwind direction, i.e. the grid spacing increases from left to right for example $\xi_{k}=[-103 / 4]$. In Figure 23 (left) the stability eigenvalues for this case are shown together with the stability region for the 4th order Runge-Kutta time integration scheme. The opposite case i.e. stretching in the downwind direction with $\xi_{k}=([-3 / 401])$ is also shown in Figure 23 (right). As can be seen the upwind stretching, together with the centered stencil is unconditionally unstable for explicit time-stepping schemes. 

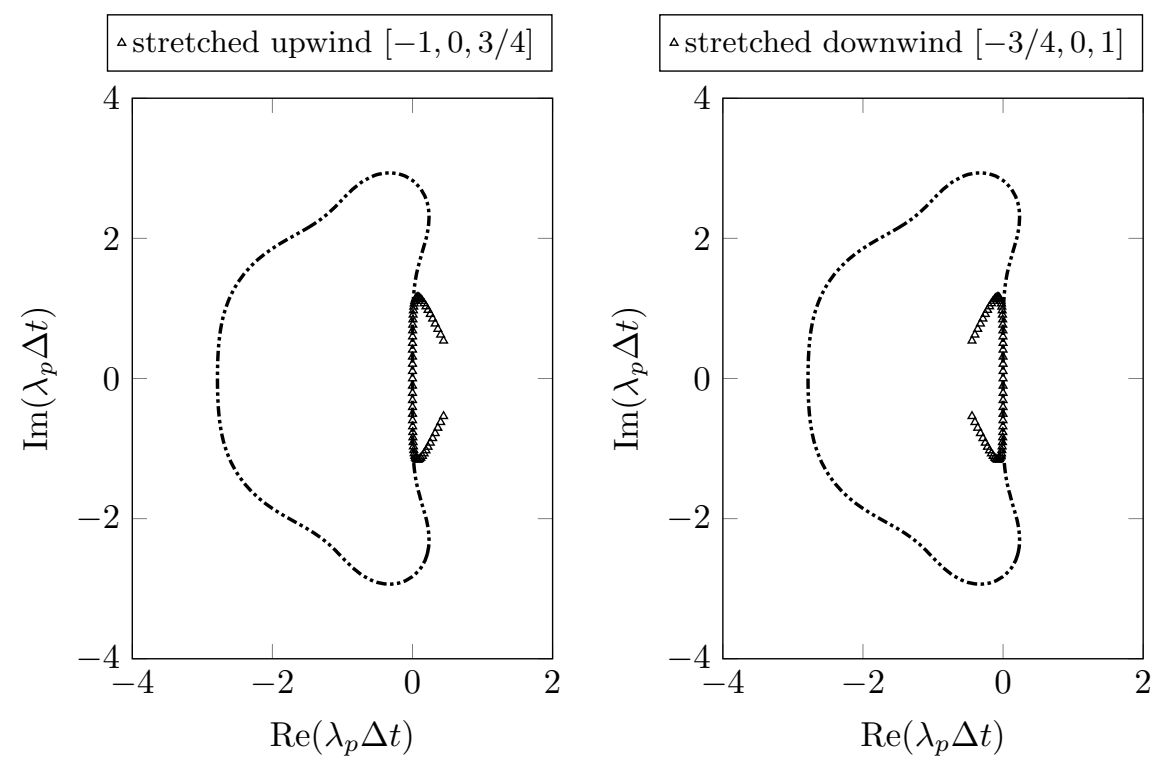

Figure 23: Eigenvalues for two stretched grids from a von Neumann stability analysis. (Left) upwind stretching, and (right) downwind stretching. The wave propagates from left to right. 